\title{
Etnobotánica de los indigenas Ulwas del Suroriente de Nicaragua y Comparaciones con el Conocimiento Botánico de los Miskitos
}

\author{
Félix G. Coe y Gregory J. Anderson
}

Los sumus o mayangnas (sumu-mayangnas) son uno de los grupos amerindios del suroriente de Nicaragua (Conzemius 1932; Hale y Gordon 1987; Nietschmann 1969; Williamson, Avilés, y McLean 1993). Históricamente conformaban cinco subgrupos: panamahka, twahka, ulwa, bawihka y kukra (nombres derivados del lenguaje local). Los dos últimos ya no existen (Conzemius 1932; Romero et al 1992). En la actualidad, los tres subgrupos que existen son identificados, principalmente, por sus características lingüísticas (Hale 1991; McLean 1996; Noewood 1997). Los sumu-mayangnas del norte hablan pamahka y twahka; y los del sur, ulwa, pero el $62 \%$ de sus respectivos vocabularios básicos es compartido por las tres lenguas (CIDCA 1989; Hale 1991). Los lenguajes de los sumu-mayangnas están históricamente emparentados al lenguaje miskito, y pertenecen al grupo de las lenguas misumalpas las cuales son ahora estructuralmente idénticas (CIDCA 1985; Hale 1991; McLean 1996; Norwood 1997). Los panamahkas y los twahkas viven hoy en lo que se conoce como la Región Autónoma Atlántico Norte (RAAN); y los ulwas, en la Región Autónoma Atlántico Sur (RAAS), una división subpolítica de la antigua región del departamento de Zelaya (Hale y Gordon 1987; Williamson, Avilés, y McLean 1993) (Figura 1). La población de los sumumayangnas, en el oriente de Nicaragua, está estimada entre 7000 a 11000 habitantes: $73 \%$ son panamahka, $16 \%$ son twahka, y solo el 11\% son ulwas (CIDCA 1982; Hale and Gordon 1987; Williamson, Avilés, y McLean 1993). El territorio de los ulwas consiste de pequeños asentamientos en las tierras bajas de los pantanos y bosques a lo largo de las orillas del río Grande de Matagalpa, río Kurinwas, y rio Wawashang.

De los tres subgrupos en el oriente de Nicaragua, los ulwas son los más aculturados. Esto se debe al continuo contacto con forasteros por más de un siglo, mayormente con misionarios y comerciantes (Bell 1989; Conzemius 1932; Dozier 1985; Nietshmann 1979; Roberts 1827; Romero 1995; Smutko 1985). El estudio del conocimiento de las plantas por parte de los ulwas se ha hecho más urgente, por la amenaza de que pueda desaparecer por completo a causa de los miskitos y la cultura de los mestizos (de descendencia indígena y europea respectivamente). Una manifestación auxiliar de aculturación puede convertirse en una perdida irremplazable de sabiduría botánica existente desde hace cientos de años. Por fortuna, este tipo de sabiduría es uno de los elementos de las culturas que se tarda más en desaparecer (Boom 1987; Schultes 1990). Sin embargo, sin la propia documentación se corre el riego de que desaparezca por completo.

Estudios etnobotánicos de los sumu-mayangnass han sido limitados a reportes de ciertos grupos de plantas medicinales por parte de Barrett (1994) y Fey y Sindel (1993). Otros estudios botánicos que incluyen material del oriente de Nicaragua son de Dennis (1988), Salter (1950), Seymour (1980), y Taylor (1962, 1963). Sin embargo, éstos solo incluyen descripciones generales del uso de plantas en el área. Estudios exhaustivos etnobotánicos que se han concentrado en el uso general de plantas por grupos indígenas del oriente de Nicaragua han sido realizados por Coe (1994) y Coe y Anderson (1996a, 1997).

Nuestro estudio fue restrictamente enfocado en la etnobotánica de los ulwas del suroriente de Nicaragua, el grupo más pequeño de los tres subgrupos. La inestabilidad política y los serios riesgos en el área de estudio restringieron las visitas al norte del país donde se encontraban los otros grupos sumu-mayangnass (Ver Figura 1). Debido a su proximidad geográfica y 
su larga historia de interacción cultural (Conzemius 1932. Nietschmann 1969; Roberts 1827), fue necesario hacer comparaciones entre los sumu-mayangnass y los miskitos (Coe y Anderson 1997). Las especies medicinales fueron el enfoque principal en estas comparaciones. Las especies nativas fueron el principal enfoque debido a que sus usos han sido menos influenciados por otras culturas, por lo cual se facilita el estudio y se hace más informativo hacia la historia y contacto cultural.

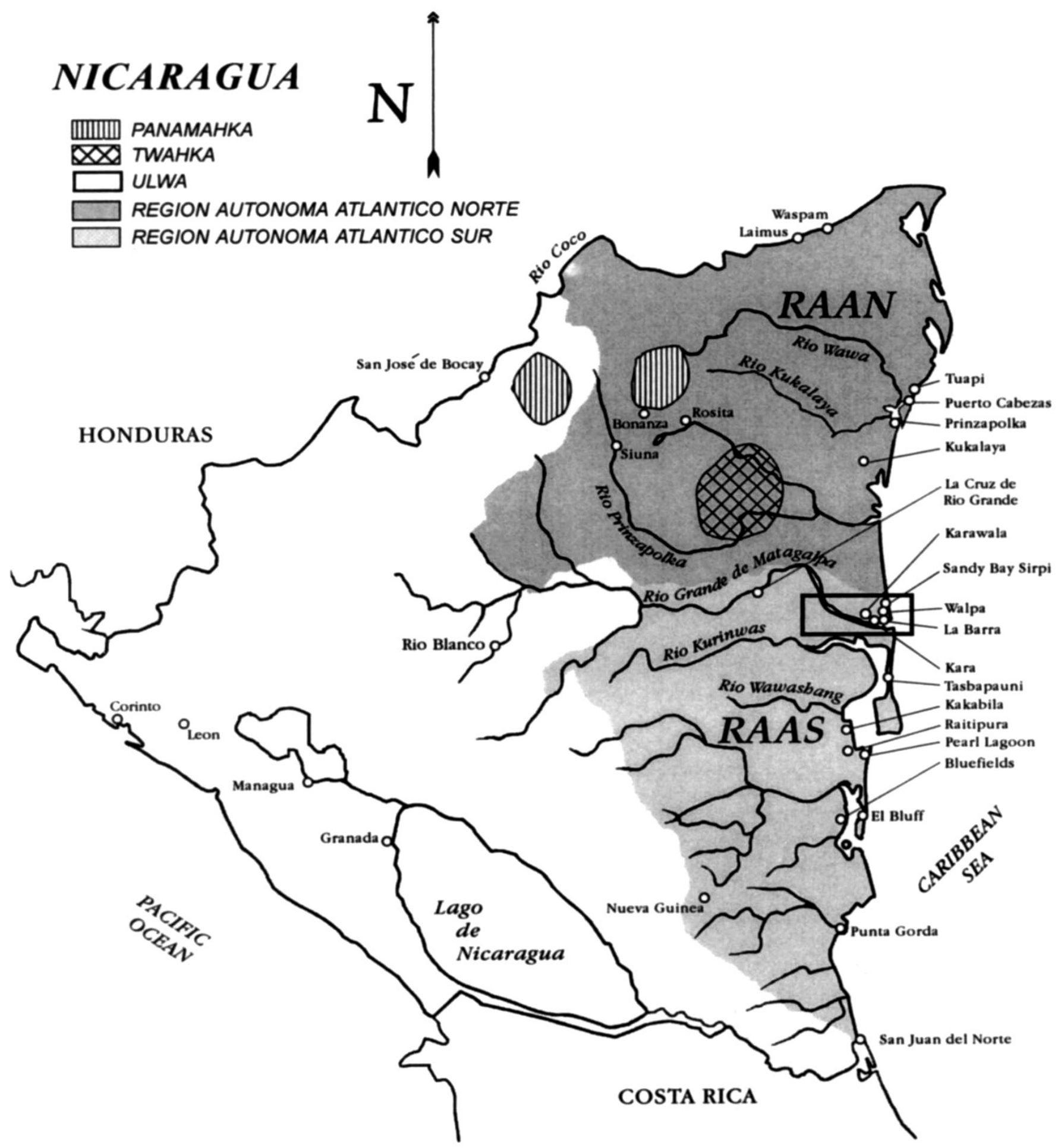

Fig.1 Mapa de Nicaragua que muestra los asentamientos ulwas en el oriente de Nicaragua y las áreas de estudio (localizadas en rectángulos). 


\section{AREA DE ESTUDIO}

El territorio de los ulwas se encuentra en el suroriente de Nicaragua entre $12^{\circ} 50^{\prime}$ a $13^{\circ} 00^{\prime}$ latitud Norte y $83^{\circ} 00^{\prime}$ a $84^{\circ} 00^{\prime}$ longitud Occidente (Figura 1). Las elevaciones en esta área varían desde nivel del mar hasta $200 \mathrm{~m}$. El enfoque del área de estudio fue principalmente en Kara y Karawala, los dos asentamientos más grandes de los ulwas en el suroriente de Nicaragua, los cuales comparten territorio con los miskitos. Karawala es el más grande de los dos grupos, con 1,200 habitantes; Kara solo tiene 30 habitantes que hablan la lengua ulwa (observación personal de Coe 1992, 1993, 1997, Hale 1991; Williamson, Avilés, y McLean 1993). El clima en esta región es tropical con temporadas de lluvia de seis a ocho meses y temporadas de sequía no muy bien definidas. El promedio de la precipitación anual es de 2000 a 4000mm (aumentando de norte a sur), y el promedio de la temperatura es de 25 a $30^{\circ} \mathrm{C}$ (Incer 1975).
El ecosistema predominante en esta área es de bosques perennes y de hojas anchas (de tierra firme en los bosques tropicales húmedos y de pantanos) y en ocasiones áreas de sabanas con pinos. Algunas de las especies dominantes en estos bosques son cedro español (113 Cedrela odorata [los números son guías para encontrar las especies en el Apéndice que también incluye los nombres ulwas, autoridades y familias]), caoba (114 Swietenia macrophylla), sambogum (48 Symphonia globulifera), samwood (36 Cordia alliodora), y Santa María (46 Calophyllum brasiliense). Las especies de pinos dominantes en las sabanas son saw cabbage palm (184 Acoelorraphe wrightii) y pino caribeño (4 pinus caribaea). Se estima que 8,000 especies de plantas vasculares existen en Nicaragua (observación personal de Stevens como parte del proyecto de la flora de Nicaragua). Coe y Stevens (comunicación personal). Este proyecto explica la distribución de especies en Nicaragua: cerca de 3,500 especies crecen en el oriente de Nicaragua, 2,000 en el nororiente (RAAN) y 3,000 en el suroriente (RAAS).

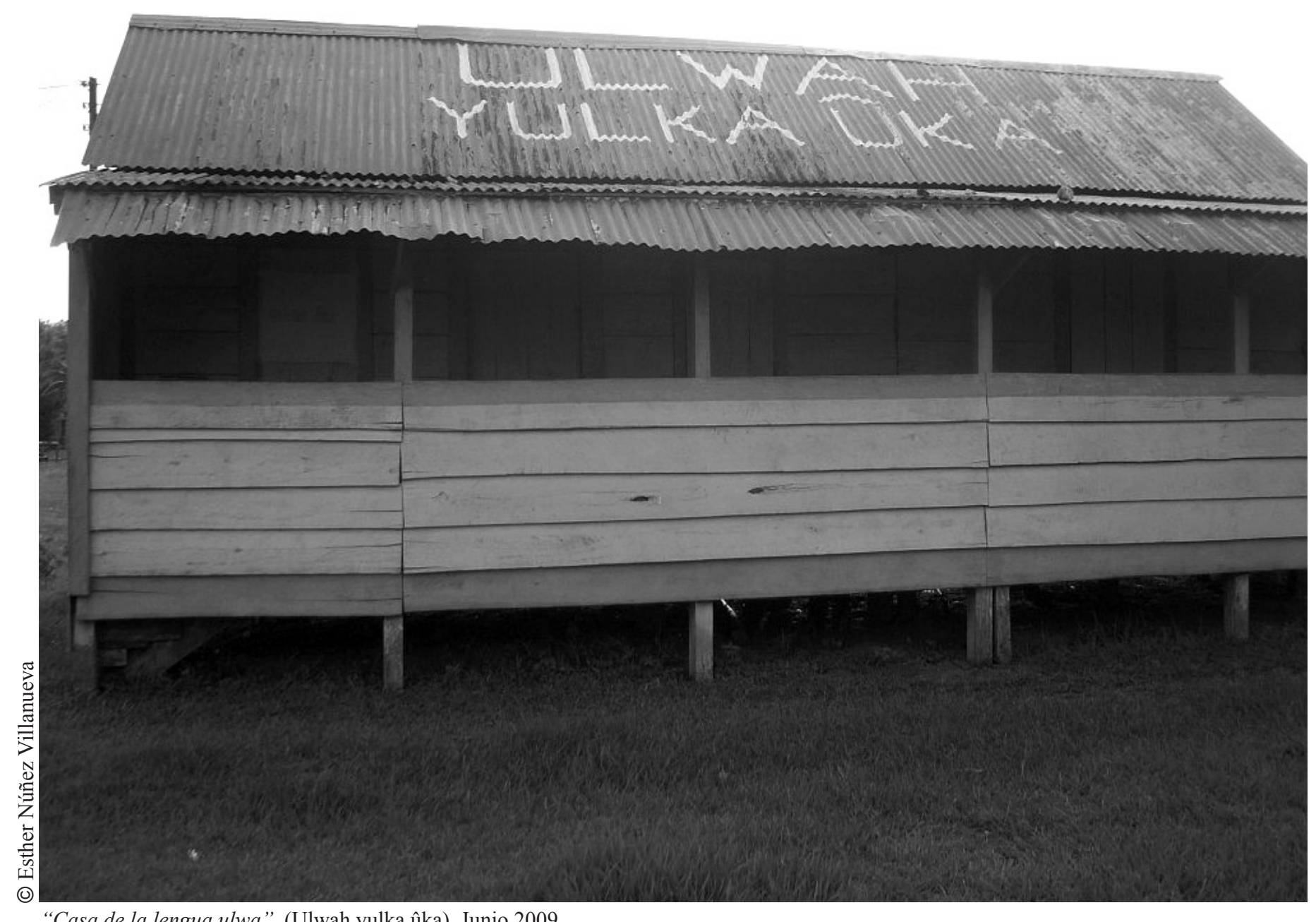

“Casa de la lengua ulwa”. (Ulwah yulka ûka), Junio 2009. 
TABLA 1. Plantas utilizadas por los ulwas organizadas por rango taxonómico y usos

\begin{tabular}{|c|c|c|c|c|}
\hline & Comida & Medicinal & Otros & Total \\
\hline Familias & 38 & 69 & 36 & 72 \\
\hline Genero & 57 & 146 & 71 & 174 \\
\hline Especies & 69 & 187 & 84 & 225 \\
\hline
\end{tabular}

TABLA 2. Origen de todas las especies utilizadas por los ulwas.

\begin{tabular}{|c|c|c|}
\hline Condición & Total & \% \\
\hline Salvajes & 174 & 77 \\
\hline Domesticadas & 30 & 14 \\
\hline Semi-domesticadas & 9 & 4 \\
\hline Compradas & 12 & 5 \\
\hline Total & 225 & \\
\hline
\end{tabular}

\section{METODOS}

La información y las muestras recogidas durante los estudios (mayo a julio 1992, diciembre a enero 1992/1993, mayo a julio 1993, diciembre 1997). Los viajes fueron planeados con el fin de obtener material valioso y evitar cualquier tipo de duda con las muestras obtenidas. El área de estudio consistió en la recolección de muestras de plantas durante viajes $\mathrm{y}$ entrevistas con practicantes (yerberos, chamanes, y parteras) de edades entre 40 a 65 años. Las entrevistas se realizaron con las técnicas empleadas por Coe (1994) Y Coe y Anderson (1996a, 1997). Las entrevistas se condujeron en el lenguaje criollo, ulwa y español. Un intérprete fue usado para llevar a cabo las entrevistas en ulwa. El marco de trabajo fue dividido en dos fases: 1) La información obtenida se guardaba en una hoja de datos, notas, grabaciones de audio para documentar el uso de las plantas y recolectar, y una lista de especies; 2) viajes con los practicantes para recoger muestras de las plantas. La información etnobotánica/ especies colectadas fueron obtenidas en los pueblos de Kara, Karawala, y en las aldeas a lo largo del río Grande de Matagalpa, río Kurinwas y río Wawashang, en el suroriente de Nicaragua (Figura 1). Para facilitar el proceso de comparación en el uso de plantas entre los ulwas y los miskitos (dividido en dos grupos: los miskitos del norte (RAAN) y miskitos del sur (RAAS), (Figura 1). Las muestras que se colectaron durante el viaje están ahora depositadas en el Herbario de la
Costa Atlántica de Nicaragua, y sus duplicados se encuentran en el Jardín Botánico de Missouri (MO) y la Universidad de Connecticut (CONN). Los especímenes fueron identificados por los autores y especialistas de varios herbarios los cuales se encuentran listados en los agradecimientos. La metodología usada para verificar los nombres de las localidades, los nombres comunes de las plantas, los nombres de su compuesto orgánico, y el análisis bioquímico de las plantas se encuentra en Coe (1994), Coe y Anderson (1996a,b, 1997), Green (1997), Guerrero y Soriano de Guerrero (1985), e Incer (1985).

\section{RECURSOS DE PLANTAS}

La taxonomía de los ulwas proviene de diversos grupos de plantas distribuidos entre 225 especies, 174 géneros, y 72 familias (Apéndice y Tabla 1). Las especies usadas incluyen plantas salvajes, semidomesticadas, y domesticadas (Tabla 2). Los ulwas obtienen las plantas por medio de sus cultivos, mercados (para "plantas compradas" Tabla 2), jardines y el bosque. Cerca de 187 especies son medicinales, 69 son alimenticias, y 84 son para uso auxiliar, como ropa, construcción y artesanías (Tabla 1). Las plantas en la categoría de varios usos juegan un papel importante, ya que proveen a los ulwas con los materiales necesarios para su subsistencia, tratamientos medicinales y hasta ingreso económico. 


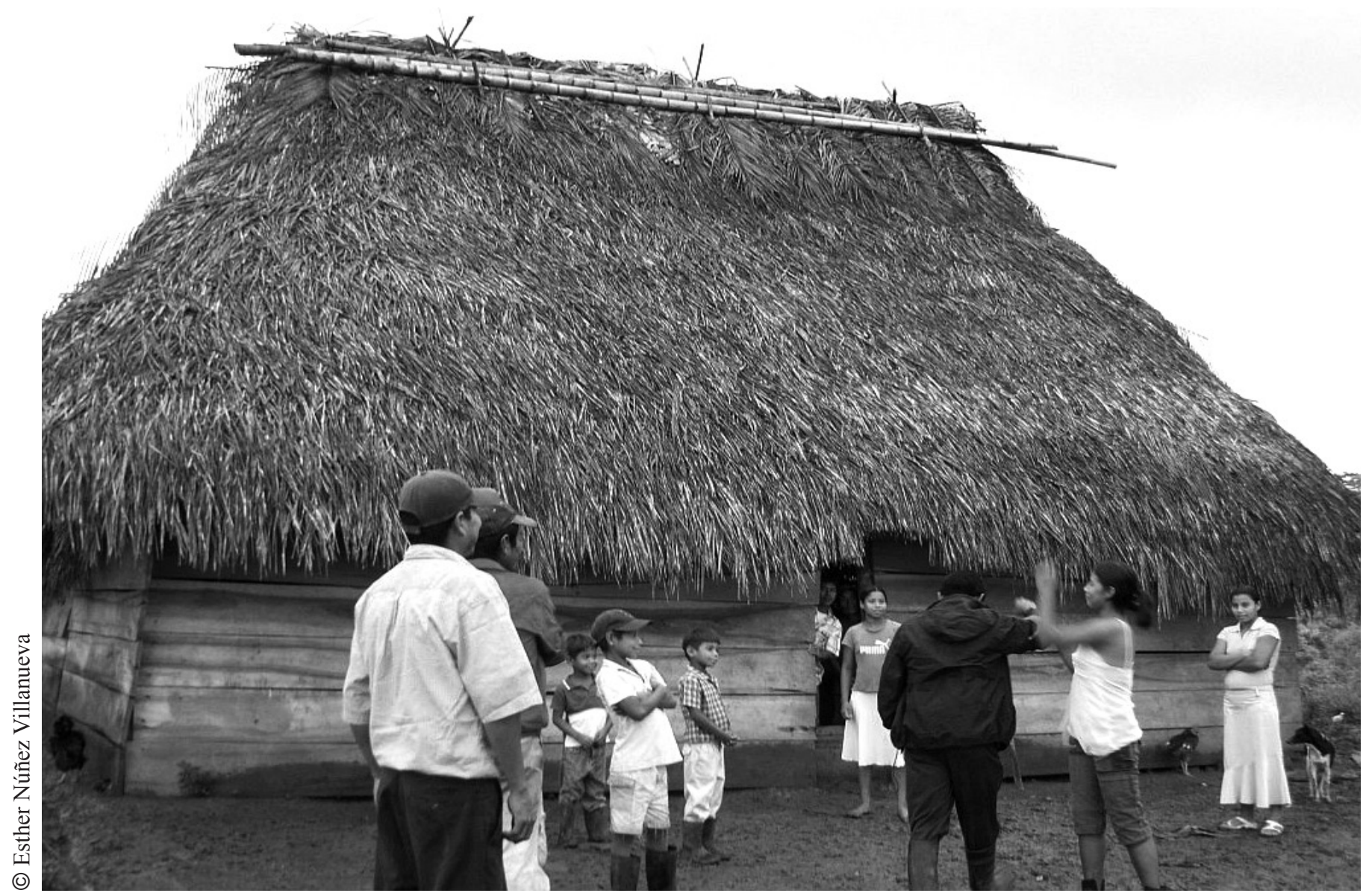

Elaboración de ropa y construcción de artesanias, Junio 2009.

TABLA 3. Especies de plantas alimenticias de los ulwas, organizadas por origen y condición. Números utilizados en esta y otras tablas demuestra la cantidad de especies utilizadas.

\begin{tabular}{|c|c|c|c|c|c|c|}
\hline Origen & Domesticadas & Compradas & $\begin{array}{c}\text { Condición } \\
\text { Semi-Domesticadas }\end{array}$ & Salvajes & Total & $\%$ \\
\hline Nativas de Nicaragua & 6 & 0 & 3 & 20 & 29 & 42 \\
\hline Introducidas & 24 & 9 & 6 & 0 & 39 & 57 \\
\hline Naturalizadas & 0 & 0 & 0 & 1 & 1 & 1 \\
\hline Total & 30 & 9 & 9 & 21 & 69 & \\
\hline$\%$ & 43 & 13 & 13 & 31 & & \\
\hline
\end{tabular}

\section{CULTIVOS}

Los cultivos de los ulwas conocidos como yâmak son el principal recurso para su alimentación básica. Los cultivos de alimentos son sembrados en áreas de una a dos hectáreas en que se practica el método de tala y quema. Ciertas áreas de los bosques son seleccionadas para ser despejadas al comienzo de la temporada de sequía (marzo a abril); durante este proceso, las especies de árboles útiles (banak [121 Virola Koshnyi], bittawood [155 Quassi amara], hog plum [9 Spondias mombin], sanwood [36], cedro español [113]) se mantienen a salvo y son protegidos para no ser quemados. La selección de las especies a proteger es determinada por su valor como recurso de alimento, medicina, madera, forraje u otros usos. Las creencias religiosas también juegan un papel importante en la selección de las especies de 
árboles que deben ser protegidos; en el caso de kapok (34 Ceiba pentandra), éste es protegido porque se cree que los espíritus viven dentro de este árbol. Luego que la vegetación seca es removida, la tierra en donde se va a cultivar es quemada (usualmente a finales de abril o principios de mayo) para permitir la siembra antes de que la temporada de lluvias comience a finales de mayo.

La siembra se realiza con instrumentos para tal fin (1.5 a $2.0 \mathrm{~m}$ de largo) o con un azadón (macana). Todos los miembros de la familia participan en el cultivo, mantenimiento y cosecha. La mayoría de las especies alimenticias usadas por los ulwas son especies de cultivos anuales (fríjoles [94 Phaseolus vulgaris], maíz [222 Zea mays], arroz [216 Oryza sativa]) intercalados con los cultivos perennes (banano [204 Musa paradisiaca var. Sapientum], cacao [165 Theobroma cacao], yuca [71 Manihot esculenta]). La mayoría de los cultivos de plantas alimenticias de los ulwas son de especies exóticas de los trópicos del Nuevo Mundo (NM) y del Viejo Mundo (VM) (Tabla 3).

Los ulwas cultivan un total de 30 especies domesticadas para el consumo local (Tabla 3). Los cinco cultivos más importantes ulwas son banano (204), frijoles (94), yuca (71), maíz (222), y arroz (216). Los sumu-mayannas dependen mayormente del maíz (222) comparados con los miskitos, los Rama, y los Garífuna. Sin embargo, de los tres subgrupos sumu-mayannas, los ulwas son el grupo que menos depende del maíz (222) como alimento básico. Este papel tan importante del maíz (222) en la dieta de los otros dos subgrupos se debe en parte a factores históricos (ya que estos dos han estado en más contacto con la población mestiza); además de que la tierra en que viven es más adecuada para el cultivo de maíz (222). La mayoría de la tierra disponible de los Ulwa es tierra húmeda; la cual es más adecuada para el cultivo de yuca (71) y arroz (216). Estos cultivos también son fructuosos porque la yuca (71) puede crecer en suelos pobres, y el arroz (216) a lo largo de las orillas de los ríos. Otros cultivos de menos importancia incluyen la piña (191 Anana comosus), plátano (203 Musa paradisiaca), caña de azúcar (221 Sacharum officinarum), y guineo cuadrado (202 Musa sp.).

\section{MERCADOS}

De 225 especies usadas (Tabla 2) por los ulwas solo 12 de ellas son compradas en los mercados. Estas plantas son de origen del Nuevo Mundo [NM] y del Viejo Mundo [VM], entre ellas se encuentran azafrán (224
Curcuma longa[VM]), canela (100 cinnamomum zeylanicum [VM]), clavos (123 Syzygium aromaticum [VM]), jengibre (225 Zingiber officinale [VM]). Ajo (201 allium sativum [VM]), nuez moscada (120 Myristica fragans [VM]), cebolla (200 allium cepa [VM]), mani (74 Arachis hypogaea [NM]), papa (162 Solanum tuberosum [NM]), y tabaco (159 Nicotiana tabacum $[\mathrm{NM}])$. En los mercados no se encuentran plantas nativas del territorio ulwa. La mayoría de las especies compradas en los mercados son especies usadas como aliños o condimentos (7 de 12 especies). Cuándo y cómo los aliños y condimentos fueron introducidos entre los ulwas es desconocido. Se sospecha que, durante un largo periodo, estas especies fueron traspasadas a los ulwas por los miskitos los cuales las obtuvieron durante el contacto con europeos y los ingleses (Coe y Anderson 1997).

\section{HUERTAS}

La mayoría de los hogares ulwas tienen huertas en donde cultivan especies alimenticias y medicinales. Algunas de las especies alimenticias que se cultivan en estos huertos incluyen banano (204), (115 Artocarpus altilis), ñame de coco (183 Xanthosoma sagittifolium), malanga (182 Colocasia esculenta), guineo cuadrado (202), pimientos ([156 Capsicum annuum var. Glabriusculium], [157 C breadfruit. Chinensis], [158 C. Frutescens $]$ ), plátano (203), y yampee (198 Dioscorea trifida). Otras especies populares que se encuentran en estas huertas son aguacate (101 Persea americana), anacardo (7 Anacardium occidentale), drap (125 Passiflora quandragularis), guayaba (122 Psidium guajava), hog plum (9), kinep (147 Melicoccus bijugatus), mango (8 Mangifera indica), soursoup (12 Annona muricata), y star apple (149 Chrysophyllum cainito).

Algunas especies medicinales también son sembradas en estas huertas, y son usadas generalmente para el tratamiento de enfermedades comunes como la tos, fiebre, y dolor. Algunas de las especies medicinales más cultivadas en estos huertos son barsley (99 Ocimum Micranthum), christmas blossom (75 Cassia alata), culantro (14 Eryngium foetidum), drap (125), fever grass (210 Cymbopogon citrus), ghost bush (5 Blechum brownei), guinea hen (126 Petiveria alliacea), kiskita (65 acalypha arvensis), ram goat dash along (168 Turnera ulmifolia), tree of life (61 Kalanchoe pinnata), arroz salvaje (154 Scoparia dulcis), wî wî (172 Lantana trifolia), y worm bush (102 Spigelia anthelmia). 


\section{BOSQUES}

Los bosques son la fuente más importante de material medicinal para los ulwas, pues, de ellos provienen todas las especies salvajes presentes en la Tabla 2. Las plantas salvajes son usadas como suplementos alimenticios, medicinas y para otros fines como construcción, artesanías, colorantes y taninos. Las plantas alimenticias obtenidas de los bosques son el 31\% de las especies alimenticias de los ulwas (Tabla 3). En contraste con las especies domesticadas, la mayoría de las especies alimenticias usadas por estos indígenas son nativas de Nicaragua (20 a 21 especies) (Tabla 3); sin embargo, éstas solo sirven como fuente de especies alimenticias. Las especies encontradas en los bosques son las más importantes como fuente de especies medicinales para los ulwas. La mayor parte de las plantas usadas en su farmacopea son plantas salvajes (139 de 187 especies, ver Tabla 4). Las especies de plantas del bosque son generalmente usadas para usos auxiliares (84 de 225 especies; ver Tabla 1) y son de importancia para los ulwas, porque de ellas obtienen madera (14 de 84 especies) para uso local o para la venta. La venta de madera aún continúa siendo una fuente importante de ingresos para los ellos. Son pocas las áreas de árboles de madera que aún existen (pino caribeño [4]) como resultado de la gran explotación que comenzó a principios de siglo y que continuó hasta finales de 1970. Por lo cual, solo la madera nativa (caoba [114], nancitón [68 Hyeronima alchorneoides], saba [112 Carapa guainensis], sambogum [48], samwood [36], Santa María [46], cedro español [113]) son cultivadas.

Tabla 4. Especies medicinales de los ulwas, organizadas por origen y condición

\begin{tabular}{|c|c|c|c|c|c|c|}
\hline Origen & Domesticadas & Compradas & $\begin{array}{c}\text { Condición } \\
\text { Semi-Domesticadas }\end{array}$ & Salvajes & Total & $\%$ \\
\hline Nativas de Nicaragua & 6 & 0 & 3 & 138 & 147 & 79 \\
\hline Introducidas & 21 & 11 & 7 & 1 & 40 & 21 \\
\hline Naturalizadas & 0 & 0 & 0 & 0 & 0 & 0 \\
\hline Total & 27 & 11 & 10 & 139 & 187 & \\
\hline$\%$ & 15 & 6 & 5 & 74 & & \\
\hline
\end{tabular}

\section{USOS DE PLANTAS}

Las plantas usadas por los ulwas hacen parte de una larga variedad de géneros y familias. Al igual que los garífunas ( Coe 1994; Coe y Anderson 1996a) y los miskitos (Coe y Anderson 1997), la categoría de plantas más usada incluye la de especies medicinales. Dos estudios hechos anteriormente han descrito el uso de especies medicinales entre los sumu-mayannas (Barrett 1994, y Fey y Sindel 1993). La mejor descripción previa del uso de especies medicinales por los sumu-mayannas fue escrita por Conzemius (1932). Sin embargo, después del estudio de Conzemius, muchos cambios han ocurrido dentro de la cultura de los sumu-mayannas. El conocimiento de las plantas de los ulwas ha sido gravemente afectado a causa de la toma de nuevas costumbres, en especial afectando el conocimiento de especies de plantas usadas para construcción, artesanías, vestido, comida, y medicina. Estas categorías se discuten a seguir.

\section{VESTIMENTA}

Antes de la llegada de los misioneros al oriente de Nicaragua a finales de 1800, la ropa usada por los ulwas consistía en túnicas de tela de corteza (kahlau) con un agujero grande para la cabeza y un lazo atado a la cintura (Conzemius 1932). La kahlau era hecha con fibras de corteza interna de higo salvaje (118 Ficus sp.) y tunu (119 Poulsenia armata) (Conzemius 1932). Aunque los ulwas contemporáneos usan ropa moderna, la cual obtienen de comerciantes o en los mercados, aún siguen usando fibras naturales para fabricar ropa y artesanías (Fig 2,3).

La fibras naturales más usadas son obtenidas de la corteza de mahoe (109 Hibiscus tiliaceus), sulduih (166 Heliocarpus donnollsmithii), tunu (119), higo salvaje (118), y pino salvaje (192 Bromelia pinguin). Las fibras de estas especies son tratadas con jugo de limón (144 Citrus aurantifolia) para reducir la oxidación y aligerar los colores. Luego las fibras son sumergidas en un 
baño o pintadas con una combinación de los siguientes colorantes naturales: negro, de sambogum (48); café, de caoba (114); gris, de kerosín (53 Terminalia oblonga); anaranjado, rojo y amarillo, de annato (33 Bixa orellana); rojo, de withes (31 Arrabidaea chica); y amarillo y rosado, de azafrán (224). Su vestimenta es muy simple, y los ornamentos personales son pocos los cuales consisten mayormente de collares y amuletos hechos de lágrimas de Jobs (209 Coix lacrymajobi), maklala (88 Dioclea megacarpa), wabala (92 Mucuna urens), y conchas de mar. El calzado es generalmente usado para ir a la iglesia o para visitarlos asentamientos mestizos.

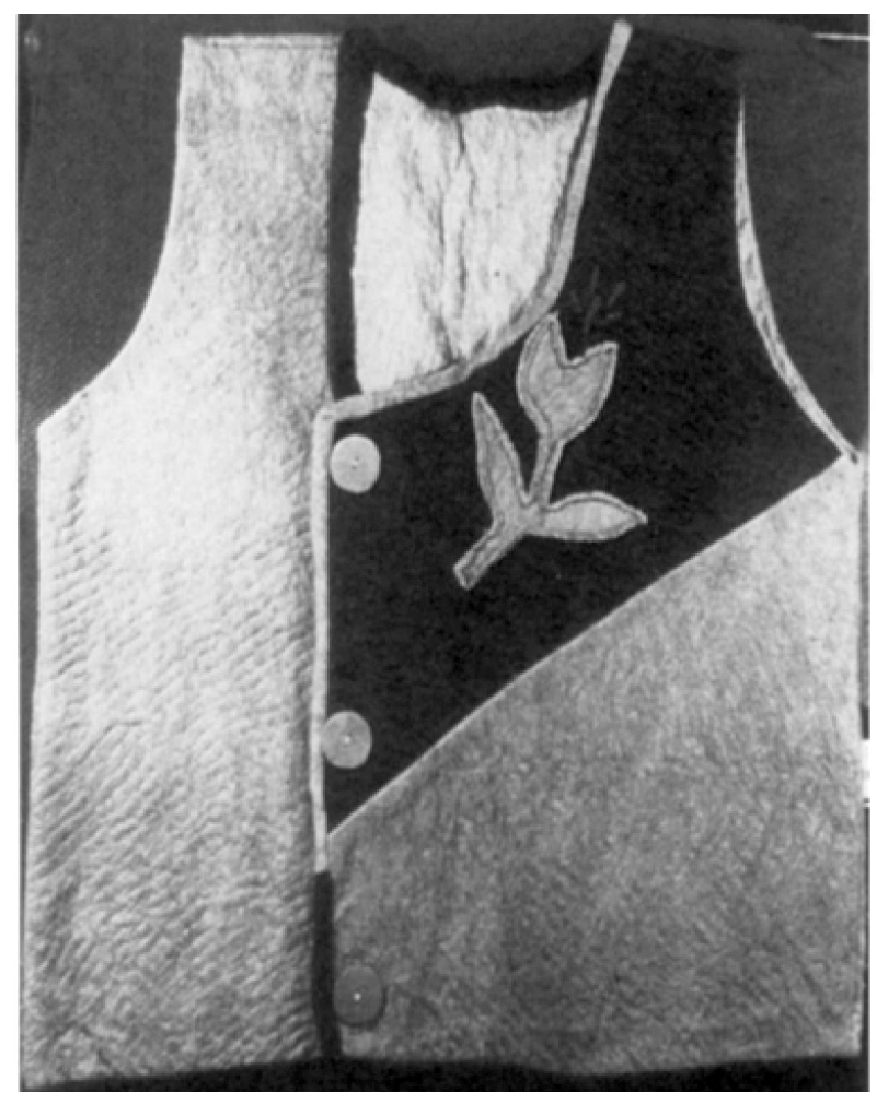

Fig. 2. Chaleco con tela de la corteza obtenida de Tunu (Poulsenia armata, Moraceae).

\section{CONSTRUCCION Y ARTESANIAS}

Los ulwas usan materiales tradicionales y materiales modernos, para la construcción (Figura 4) y fabricación de artesanías. Los materiales modernos (colorantes, fibras, puntillas, y láminas de metal) usados para la construcción y artesanías son obtenidos por medio de comerciantes o en las tiendas rurales. Los materiales tradicionales usados para la construcción y artesanías son obtenidos de 84 especies.

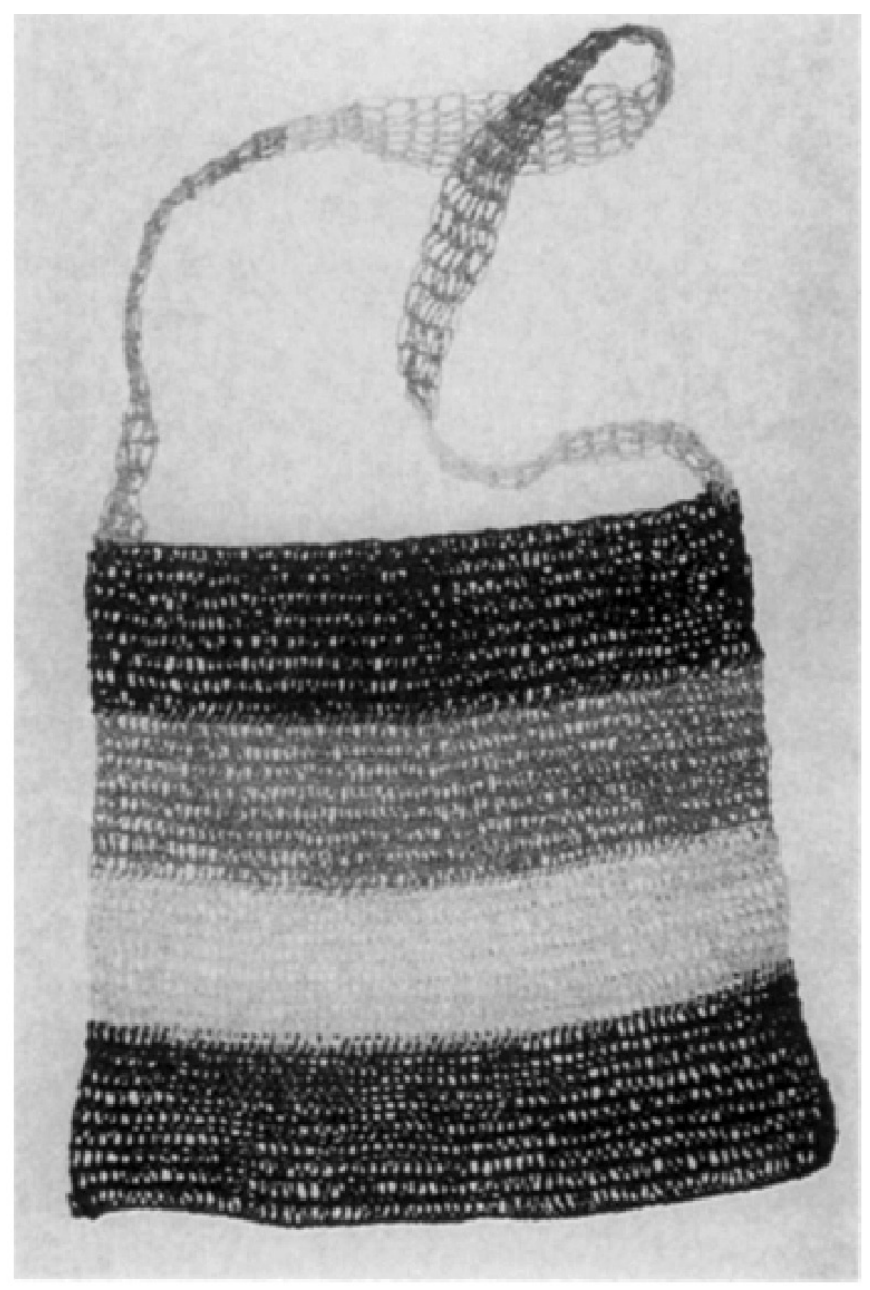

Fig.3. Bolsa ulwa hecha con fibras de sulduih (Heliocarpus donnell-smithii, Tiliaceae) y pintada con extractos de pan lalahka (Chlorophora tintoria, Moraceae, limnah (Terminalia oblonga, Combretaceae), y azafrán (Curcuma longa, Zingiberaceae)

La mayoría de las casas consisten en una habitación para dormir, cocina, cuarto para el almacenamiento de los granos, y un área en donde se crían los animales. Los materiales usados en la construcción de casas son obtenidos de 35 especies. Las seis especies más usadas son pino caribeño (4), caoba (114), sambogum (48), samwood (36), Santa María (46), y cedro español (113). Algunas de las especies tienen usos específicos. Las paredes y los techos son sostenidos con cepa de guacu (18 Aristolochia trilobata), mahoe (109), y withes (31). Los pilotes son hechos con especies resistentes como ebo (89 Dipteryx oleifera), oaka (150 Manilkara zapota), y sipul (151 Pouteria sapota). Los techos son hechos con hojas de palma como hone (188 Elaeis guineensis), saw cabbage palm (184), y sconfra (186 Calyptrogene 


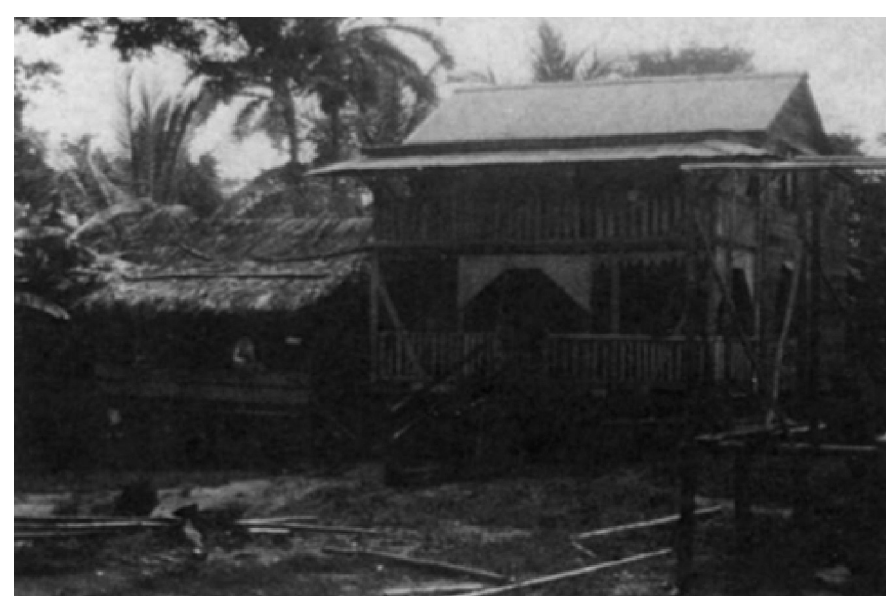

Fig. 4. Casa ulwa fabricada con una combinación de materiales de construcción tradicionales y modernos.

ghiesbreghtiana), y con hojas de metal galvanizado. Las paredes son hechas con bambú (208 Bambusa vulgaris), banak (121), pino caribeño (4), saba (112), saw cabbage palm (184), y wild cane (212 Gynerium sagittatum). Los muebles (camas, bancas, mesas) son hechos con cualquier tipo de material disponible.
Las artesanías son hechas para tenerlas en los hogares o para la venta a los turistas. Las hamacas son comunes en las casas y son generalmente fabricadas de fibras de tunu (119) o compradas en los mercados de los asentamientos mestizos. Objetos artesanales populares incluyen chalecos hechos de fibra de tunu (119) (Figura 2), y tazones, figuras, tenedores, modelos de botes y canoas, hechos de caoba (114), cedro español (113), y palisandro ([81 Dalbergia brownei], [82 D. hypoleuca], [83 D. tucurensis]).

\section{ALIMENTOS}

De 69 especies usadas por los ulwas como alimento forman parte las especies domesticadas, semi-domesticadas y salvajes (Tabla 3). Al igual que los miskitos (Coe and Anderson 1997), los ulwas dependen de las especies domesticadas como la mayor fuente de alimento; las especies cultivadas por los ulwas son el $43 \%$ de su alimento básico (Tabla 3). El porcentaje restante (57\%) proviene de las especies semi-domesticadas, especies salvajes, y de las plantas que son compradas en huertas, bosques o mercados. Sus plantas alimenticias más importantes fueron mencionadas anteriormente en la sección de huertas.

Tabla 5. Especies de plantas medicinales usadas por los ulwas, organizadas por origen y tipo

\begin{tabular}{|c|c|c|c|c|c|c|}
\hline \multicolumn{7}{|c|}{ TIPO DE PLANTA } \\
\hline Origen & Árbol & Arbusto & Vid & Hierba & Total & $\%$ \\
\hline Nativa de Nicaragua & 49 & 5 & 22 & 71 & 147 & 79 \\
\hline Introducida & 10 & 3 & 2 & 8 & 23 & 12 \\
\hline Naturalizada & 3 & 1 & 2 & 11 & 17 & 9 \\
\hline Total & 62 & 9 & 26 & 90 & 187 & \\
\hline$\%$ & 33 & 5 & 14 & 48 & & \\
\hline
\end{tabular}

Tabla 6. Medicinales: partes de la planta usadas por los ulwas.

\begin{tabular}{|c|c|}
\hline Partes usadas & Número de especies \\
\hline Hoja & 123 \\
\hline Corteza & 40 \\
\hline Raíz & 32 \\
\hline Toda la planta & 28 \\
\hline Fruta & 24 \\
\hline Savia & 15 \\
\hline Tallo & 11 \\
\hline Flor & 2 \\
\hline Semilla & 2 \\
\hline
\end{tabular}


Tabla 7. Modo de preparación de especies medicinales usadas por los ulwas.

\begin{tabular}{|c|c|}
\hline Modo & Número de Especies \\
\hline Decocción & 167 \\
\hline Cataplasma & 36 \\
\hline Jugo & 21 \\
\hline Infusión & 16 \\
\hline Ninguno & 9 \\
\hline Baño & 4 \\
\hline Jarabe & 1 \\
\hline
\end{tabular}

Tabla 8. Modo de administración de especies medicinales preparadas por los ulwas.

\begin{tabular}{|c|c|}
\hline Modo & Número de Especies \\
\hline Oral & 167 \\
\hline Tópico & 73 \\
\hline Baño & 9 \\
\hline Inhalación & 1 \\
\hline
\end{tabular}

\section{MEDICINAS}

Para el tratamiento de las muchas enfermedades que afectan a los ulwas (disentería, malaria, mordidas de culebra) y las cuales son comunes en los hábitats de tierra baja del área tropical, estos indígenas han desarrollado una larga farmacopea en relación con las especies salvajes y las domesticadas (187 especies medicinales) (Tabla 1). La mayoría de estas especies medicinales son salvajes (74\%) (Tabla 4), y la gran mayoría son hierbas (48 \%) o árboles (33\%) (Tabla 5). La mayor parte de estas especies $(75 \%)$ contienen principios bioactivos, como alcaloides $(87 \%$ de las especies con materiales bioactivos) y glucósidos (13\%) (Ver Apéndice).

Los materiales usados en la preparación de las medicinas incluyen hojas, corteza, raíces, frutas, savia, tallos (de madera), flores, y semillas. En algunas instancias, toda la planta puede ser usada, incluyendo las raíces. La parte de la planta más frecuentemente usada son las hojas (123 especies), seguida por la corteza (40 especies) (Tabla 6). Los remedios de hierbas son preparados como cocciones, tópicos, jugos, infusiones, baños y jarabes. La mayoría de las medicinas son preparadas como cocciones (161 especies) (Tabla 7) y son administradas oralmente (167 especies) (Tabla 8). Estos resultados son similares a los resultados documentados por Coe y Anderson (1997) acerca de los miskitos.
Las 15 especies medicinales preferidas por los ulwas (las que tienen más de cinco aplicaciones diferentes) son: aguacate* (*especies compartidas entre los miskitos de sur y los ulwas) (101), balsam pear* (63 Mormordica charantia), bird bush (137 Borreria laevis), burbur (84 Desmodium adscendens), christmas blossom* (75 Cassia alata), flor de muerto (28 Tagetes erecta), frailecillo (70 Jatropha gossypiifolia), guayaba* (122), jackass bitters* (27 Neurolaena lobata), John Charles (98 Hyptis verticillata), limón (144), red scholars* (140 Hamelia patens), sorocontil (78 Cassia reticulata), Salvia salvaje (37 Cordia curassavica), y Tomillo salvaje (174 Lippia micromera). De estas 15 especies, los ulwas tienen 82 aplicaciones medicinales de las cuales 11 son únicas. En contraste con los miskitos del sur los cuales tienen 22 especies (con cinco o más aplicaciones) con 124 aplicaciones medicinales, 23 de las cuales son únicas. Siete de las especies más populares son usadas por ambos grupos. Los ulwas tienen 41 aplicaciones medicinales para estas especies (tres que son únicas), para los miskitos son 47 aplicaciones medicinales (nueve únicas).

Por lo tanto, como se puede esperar, las especies medicinales más usadas, entre estos dos grupos tienen a la postre los mismos usos. Los ulwas tratan sus enfermedades y molestias con medicina tradicional y moderna. La medicina tradicional consiste de rituales realizados por chamanes (watyu) para tratar las enfermedades sobrenaturales, y el uso de hierbas medicinales practicado 
por los herbalistas (dî basta talingka) para el tratamiento de las enfermedades naturales. Este último es considerado el principal método para el cuidado de la salud entre los ulwas. La medicina moderna, por otro lado es usada cuando la medicina tradicional no funciona. Sin embargo, la medicina moderna es usualmente costosa o inaccesible (a pesar de las mejoras en el transporte durante los años 80 , hechos con el fin de facilitar el acceso a la medicina moderna). El viajar a veces es tan difícil que en varias instancias, el paciente llega al hospital o a una clínica demasiado tarde para recibir un tratamiento efectivo.

El uso de especies medicinales por los ulwas es similar al de los miskitos, en el sentido de que es generalmente controlado por los practicantes (herbalistas y chamanes). Los practicantes protegen sus conocimientos con gran cautela; probablemente para aumentar su reputación y prestigio. Sin embargo, un saber general de las especies medicinales existe entre la población y los practicantes. En la mayoría de los casos, la gente utiliza los mismos materiales de las plantas usados por los chamanes y los herbalistas, pero los métodos de preparación y administración son diferentes.

\section{DISCUSION}

Los ulwas han conservado varios aspectos de su cultura, entre ellos su conocimiento etnobotánico, y su dependencia de las plantas como medicinas. Su aculturación es evidente por los cambios que han ocurrido, en especial durante los años ochenta. Algunos de los cambios más notables en el uso de plantas por los ulwas (empezando por los que tuvieron menos cambios) incluyen: sanación y curación, artesanías, construcción, alimentos y vestimenta. En la discusión a seguir, nos basamos en la información obtenida del Apéndice y los estudios de los garífunas (Coe 1994; Coe y Anderson 1996 a,b) y los miskitos (Coe y Anderson 1997), para poder hacer comparaciones con el uso de las plantas por los ulwas. Estos dos grupos fueron escogidos, porque tienen en común el mismo ecosistema y han estado en contacto con el mismo grupo de extranjeros.

La gran mayoría de las especies usadas por los ulwas son las mismas usadas por los miskitos (93\% de especies en común). Esto no es raro, ya que los dos grupos comparten el mismo territorio. Los ulwas y los miskitos del sur comparten más especies (91\% en común) y tienen un mayor intercambio en sus conocimientos botánicos que los que existen entre los ulwas y los miskitos del norte, con solo $51 \%$ de especies en común. Por lo tanto, el parecido en el uso de especies entre los ulwas y los miskitos del sur se debe en gran parte a su proximidad, por lo cual, ambos grupos interactúan con las mismas especies de plantas -aquellas que forman parte de las tierras bajas y los pantanos, en el área central del oriente de Nicaragua. Estos dos grupos también tienen ciertas creencias y prácticas en común (basadas en su extenso contacto e intercambio cultural), particularmente entre los habitantes de los pueblos de Kara y Karawala. Éstas se extienden a los conocimientos botánicos y rituales en los cuales los curanderos ulwas y miskitos trabajan juntos para el tratamiento de enfermedades graves.

La mayoría de las especies usadas y gran parte de la dieta de los ulwas y los miskitos depende de especies domesticadas, las cuales fueron mayormente introducidas por los europeos (Coe y Anderson 1997). Sin embargo, los miskitos utilizan más especies salvajes (37 especies) que los ulwas (21 especies), una diferencia que probablemente se refleja en la mayoría de la población miskita (la cual es 60 veces más grande que la de los ulwas), los largos territorios en que viven, y muchas veces el aislamiento en que algunos se encuentran.

Un 94\% de las especies medicinales de los ulwas son usadas por los miskitos del norte y del sur; sin embargo, solo el $61 \%$ son usadas por los miskitos del sur y por los ulwas. Èstos obtienen sus materiales medicinales en una área relativamente pequeña de la tierra baja y pantanos cerca del delta de el río Grande de Matagalpa en comparación con el territorio de los miskitos, que también cubre la misma área pero se extiende hasta el sur de Bluefields (ver Figura 1).

Cerca de tres cuartos de las especies medicinales usadas por los ulwas y los miskitos son nativas del oriente de Nicaragua, y son encontradas en los bosques. La familia de plantas más empleada para usos medicinales por los ulwas y los miskitos (Coe y Anderson 1997) incluyen: Asteraceae, Euphorbiaceae, Fabaceae, Rubiaceae, Solanaceae, y Verbenaceae (Tabla 9). Estos dos grupos comparten la preferencia por el uso de la misma parte de la planta (hojas), el método de preparación (cocción), y el modo de administración (oral). Sin embargo, el método de preparación para los remedios de hierbas es diferente. Por ejemplo, los miskitos preparan la mayoría de sus remedios tópicos remojando el material de la planta en 
una mezcla de agua y alcohol; los ulwas, solo la hierven en agua. Los miskitos (Coe y Anderson 1997) utilizan más medicinas modernas que los ulwas. Esto puede ser debido a que tienen mayor acceso y han estado en contacto con estas por más tiempo - en comparación con cualquier otro grupo indígena del oriente de Nicaragua (Coe y Anderson 1997).

Para los ulwas y los miskitos del sur, las enfermedades que pueden ser tratadas con un gran número de especies son: fiebre ( 66 ulwa $=\mathrm{u}, 86$ miskitos sur $=\mathrm{ms}$ ), dolores y molestias (60 u, $77 \mathrm{~ms}$ ), erupciones de la piel y llagas
(57 u, $100 \mathrm{~ms})$, diarrea (55 u, $70 \mathrm{~ms})$, infecciones (32 u, $48 \mathrm{~ms}$ ), mordidas y picadas (31 u, $39 \mathrm{~ms})$ y problemas digestivos (30 u, $42 \mathrm{~ms}$ ). La mayoría de especies usadas para el tratamiento de estas enfermedades puede ser atribuida a la ubicuidad de las enfermedades y su presencia durante todo el año. La mayoría de los remedios de hierbas son preparados mezclando diferentes especies. La mayoría de las especies usadas por los ulwas, al igual que los Garífuna (Coe 1994; Coe y Anderson 1996a) y los miskitos (Coe y Anderson 1997) contienen alcaloides y/o glucósidos, lo cual sugiere su efectividad medicinal.

Tabla 9. Comparación del número de especies y familias de los medicamentos más importantes para los ulwas y los miskitos del sur

\begin{tabular}{|c|c|c|}
\hline \multicolumn{2}{|c|}{ Número de Especies } \\
\hline Nombre de la Familia & Ulwa & Miskito \\
\hline Asteraceae & 11 & 16 \\
\hline Euphorbiaceae & 7 & 13 \\
\hline Fabaceae & 20 & 41 \\
\hline Rubiaceae & 8 & 20 \\
\hline Solanaceae & 7 & 11 \\
\hline Verbenaceae & 10 & 13 \\
\hline
\end{tabular}

En conclusión, los miskitos del sur utilizan más especies y tienen más aplicaciones medicinales que los ulwas. En adición, solo nueve especies medicinales son únicas de los ulwas. Sin embargo, existen algunas diferencias. Hay más de 30 aplicaciones únicas entre los ulwas (Tabla 10). Por ejemplo, aunque ambos usan Christmas blossom (75) y jackass bitters (27), para tratar varias enfermedades, solo los ulwas usan estas especies como febrífugo, y diurético respectivamente. Dado el contraste de territorio de los dos grupos, la pregunta es, ¿cómo pueden existir diferencias entre los dos? La mayoría de las diferencias, en las prácticas de uso de las plantas, se encuentran en las aplicaciones medicinales de ciertas especies en particular. Las especies pueden ser seleccionadas para ser o no ser parte de cierto grupo basado en 1) la efectividad de las especies; 2) los efectos no deseados (basado en opinión cultural); 3) la disponibilidad local (distribución desigual), y 4) los métodos de preparación o aplicación que a veces no son aceptados culturalmente. Un ejemplo es wild fig. (118): esta especie no es usada por los ulwas porque ellos creen que cualquier contacto con esta planta puede perturbar a los espíritus. Por otro lado, esta especie es de gran importancia medicinal para los miskitos. En algunos casos, las especies no usadas, o usadas con mucha cautela, para prevenir efectos no deseados como pesadillas, caída del cabello, envenenamiento y otros malestares.

Claramente existe una gran similitud entre el conocimiento botánico de los ulwas y los miskitos, especialmente con los miskitos del sur, en parte debido a su largo y continuo contacto el cual aumentó aun más durante el siglo XVIII y XIX, motivado por los comerciantes ingleses y luego por los misioneros (Conzemius 1932; Romero 1995; Smutko 1985). Sin embargo, durante los últimos cincuenta años ha habido un dramático aumento debido a un mejor acceso al área (por la construcción de vías y canales), resultando esto en la interacción de los ulwas con otros grupos. Por estas razones, estudios etnobotánicos como éste son importantes para la documentación del uso tradicional de plantas para fines científicos, pero, al mismo tiempo, para servir de referencia de la cultura indígena. 


\section{AGRADECIMIENTOS}

Este estudio fue parcialmente financiado por The National Science Foundation y la Universidad de Connecticut Research Foundation. Le agradecemos en mayor parte a la gente sumu-mayannas, en particular a Martin Crimmins, Modesta Galarza (partera), Grincilla Gamboa (partera), Dragas López, Juslin López Salazar, Santiago Paiba (Chaman), Geronimo Palmiatan (hierbero), Beatriz Salazar ( Partera), y Murphy Salazar (chaman), por haber recibido a uno de nosotros (Coe) en sus hogares y por haber compartido con él sus conocimientos etnobotánicos. La asistencia en el área de estudio de Rodney Martin es muy apreciada. También agradecemos al personal de BICU-CIDCA (Centro de Investigación y Documentación de la Costa Atlántica) y FADCANIC (Fundación para la Autonomía y Desarrollo de la Costa Atlántica de Nicaragua), en particular a Ray Hooker de FADCANIC. Numerosos especialistas ayudaron en la identificación de las muestras: William D’Arcy (MO), Rupert Barneby (NY), Gerrit Davidse (MO), James Grimes (NY), Helen Kennedy (UBC), Ronald Leisner (MO), Michael Nee (NY), Amy Pool (MO), Velva Rudd (SFV), George Schatz (MO), Warren D. Stevens (MO), Charlotte M. Taylor (MO), e Iván Valdespino (NY). Le agradecemos a Ellie DeCarli, Edward Graves, y Mary Jane Spring por la ayuda con las ilustraciones, tablas y el Apéndice, y a Maryke Schlehofer por la tabulación de datos y los comentarios en los borradores de los manuscritos. También apreciamos los tres críticos anónimos y los comentarios constructivos de Lawrence Kaplan.

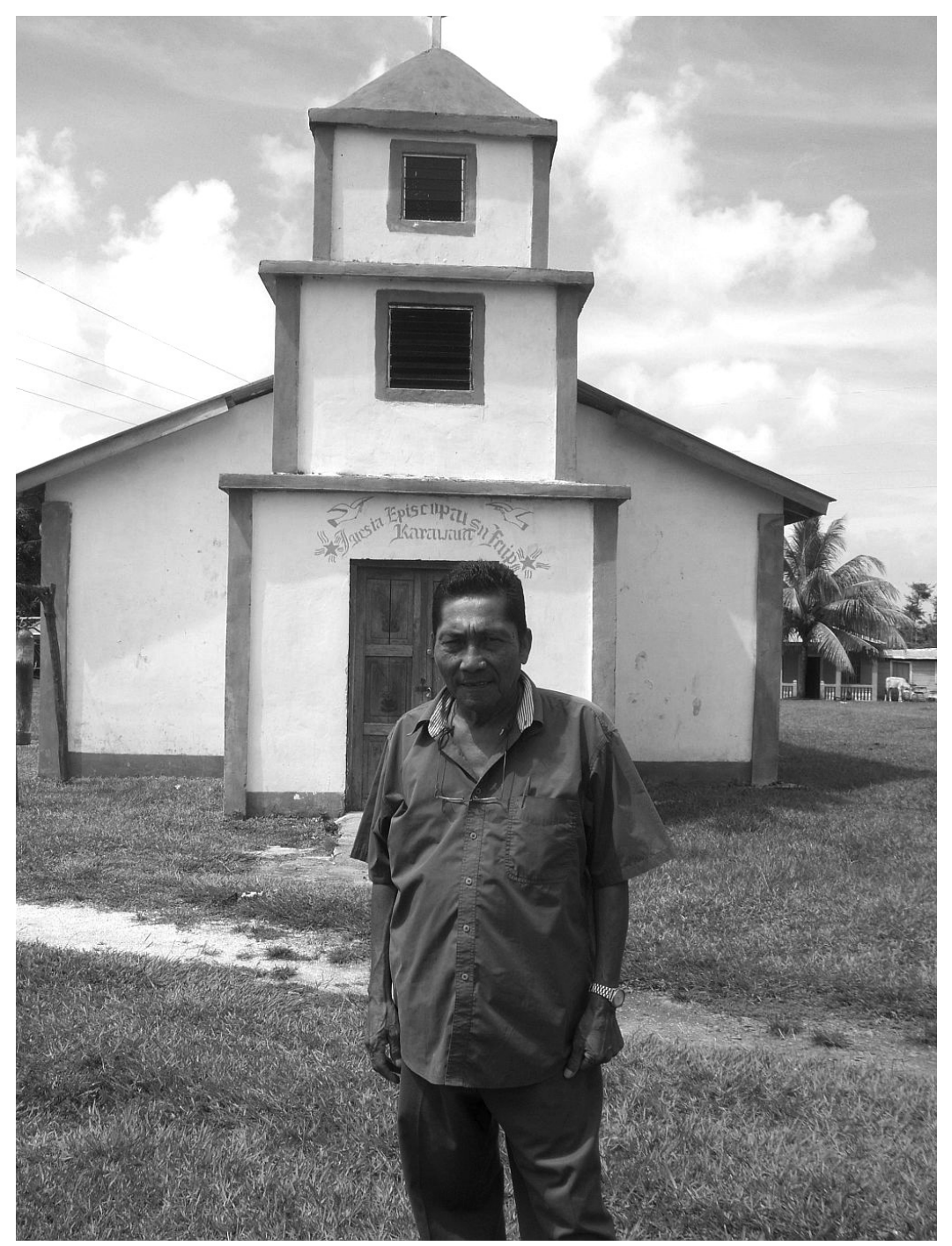


TABLA10. Aplicaciones medicinales comunes y únicas entre los ulwas y miskitos del sur. Aplicaciones medicinales: $\mathrm{A}=$ molestias y dolores; $\mathrm{B}=$ mordeduras y picaduras (serpiente, escorpión, insectos); $\mathrm{C}=$ parto y embarazo; $\mathrm{D}=$ diarrea; $\mathrm{E}=$ emético; $\mathrm{F}=$ fiebre; $\mathrm{G}=$ digestivo (dolor de estómago, úlceras, etc.); $\mathrm{H}=$ hipertensión; $\mathrm{I}=$ infecciones; $\mathrm{J}=$ diabetes; $\mathrm{K}=$ diurético; $\mathrm{L}=$ desórdenes respiratorios y pulmonares (gripa, toses, etc.); $\mathrm{M}=$ malaria; $\mathrm{N}=$ quemaduras; $\mathrm{O}=$ abortivo; $\mathrm{P}=$ gusanos y parásitos intestinales; $\mathrm{Q}=$ astringente; $\mathrm{R}=$ rituales; $\mathrm{S}=$ sarpullido y llagas; $\mathrm{T}=$ tónico y anemia (fortificante de la sangre); $\mathrm{U}=$ cortaduras y hemorragia; $\mathrm{V}=$ enfermedades venéreas; $\mathrm{W}=$ desórdenes femeninos (menstruación, hemorragia); $\mathrm{X}=$ purgante y laxante; $\mathrm{Y}=$ estreñimiento; $\mathrm{Z}=$ extracción de diente.

\begin{tabular}{|c|c|c|c|}
\hline Especies & Aplicaciones comunes & $\begin{array}{l}\text { Aplicaciones únicas } \\
\text { de los miskitos }\end{array}$ & $\begin{array}{c}\text { Aplicaciones únicas de } \\
\text { los ulwas }\end{array}$ \\
\hline Acrostichum aureum & $\mathrm{F}, \mathrm{Y}$ & A & 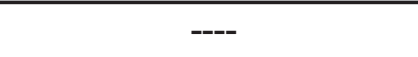 \\
\hline Aloe vera & $\mathrm{B}, \mathrm{N}, \mathrm{S}$ & $\mathrm{X}$ & ---- \\
\hline Anacardium occidentale & $\mathrm{A}, \mathrm{D}, \mathrm{S}$ & --- & $\mathrm{F}$ \\
\hline Annona glabra & $\mathrm{A}, \mathrm{C}$ & $\overline{\mathrm{L}}$ & $\begin{array}{ll}--- \\
-1\end{array}$ \\
\hline Annona muricata & C, D & $\mathrm{F}$ & ---- \\
\hline Aristolochia trilobata & $\mathrm{B}, \mathrm{G}, \mathrm{L}, \mathrm{T}$ & $\mathrm{H}$ & ---- \\
\hline Artocarpus altilis & $\mathrm{A}$ & $\mathrm{H}$ & $\begin{array}{ll}--- \\
\end{array}$ \\
\hline Avicennia germinans & $\mathrm{D}$ & ---- & Q \\
\hline Bambusa vulgaris & $\mathrm{D}, \mathrm{F}$ & $\mathrm{S}$ & --- \\
\hline Bixa orellana & $\mathrm{D}, \mathrm{L}, \mathrm{N}$ & $\mathrm{S}$ & $\begin{array}{ll}--- \\
\end{array}$ \\
\hline Borreria laevis & $\mathrm{B}, \mathrm{L}, \mathrm{S}, \mathrm{U}$ & $\mathrm{W}$ & ---- \\
\hline Carapa guianensis & $\mathrm{F}$ & $\mathrm{D}$ & A \\
\hline Cassia alata & $\mathrm{I}, \mathrm{S}, \mathrm{T}, \mathrm{X}$ & $\mathrm{D}, \mathrm{H}, \mathrm{P}$ & $F$ \\
\hline Cassia reticulata & $\mathrm{A}, \mathrm{B}, \mathrm{I}, \mathrm{S}$ & ---- & $\mathrm{W}, \mathrm{X}$ \\
\hline Cedrela odorata & $\mathrm{A}, \mathrm{F}, \mathrm{T}$ & ---- & Q \\
\hline Ceiba pentandra & $\mathrm{E}, \mathrm{K}, \mathrm{Q}$ & $\begin{array}{ll}--- \\
\end{array}$ & $\mathrm{A}$ \\
\hline Cinchona pubescens & $\mathrm{F}, \mathrm{M}$ & $\mathrm{D}$ & ---- \\
\hline Citrus aurantium & $\mathrm{D}, \mathrm{F}, \mathrm{G}, \mathrm{H}, \mathrm{I}, \mathrm{L}$ & $\mathrm{K}$ & $\begin{array}{ll}--- \\
\end{array}$ \\
\hline Connarus lambertii & $\mathrm{D}$ & Q & ---- \\
\hline Cordia curassavica & $\mathrm{A}, \mathrm{D}, \mathrm{F}, \mathrm{H}$ & ---- & $\mathrm{C}$ \\
\hline Desmodium adscendens & A, G, I, S & ---- & $\mathrm{D}$ \\
\hline Desmodium canum & A, F, I, S, V & $\mathrm{F}, \mathrm{V}$ & ---- \\
\hline Desmodium trifiorum & A, I & ---- & $\mathrm{F}$ \\
\hline Dipteryx oleifera & $\mathrm{A}, \mathrm{Q}$ & $\mathrm{Z}$ & $F$ \\
\hline Elaeis guineensis & $\mathrm{X}$ & G & $\mathrm{X}$ \\
\hline Elaeis oleifera & $X$ & G & $\mathrm{X}$ \\
\hline Elephantopus spicatus & $\mathrm{D}, \mathrm{P}$ & A & $\begin{array}{ll}--- \\
\end{array}$ \\
\hline Eryngium foetidum & $\mathrm{D}, \mathrm{G}, \mathrm{P}, \mathrm{R}$ & $\mathrm{L}$ & --- \\
\hline
\end{tabular}




\begin{tabular}{|c|c|c|c|}
\hline Especies & Aplicaciones comunes & $\begin{array}{c}\text { Aplicaciones únicas } \\
\text { de los miskitos }\end{array}$ & $\begin{array}{c}\text { Aplicaciones únicas de } \\
\text { los ulwas }\end{array}$ \\
\hline Gynerium sagittatum & $\mathrm{B}, \mathrm{I}, \mathrm{S}$ & $\mathrm{K}, \mathrm{V}$ & $\begin{array}{c}--- \\
\end{array}$ \\
\hline Hamelia patens & $\mathrm{B}, \mathrm{F}, \mathrm{I}, \mathrm{M}, \mathrm{S}, \mathrm{U}$ & $\mathrm{W}$ & ---- \\
\hline Hibiscus tiliaceus & $\mathrm{F}$ & $\mathrm{Y}$ & $\mathrm{C}$ \\
\hline Hyptis verticillata & $\mathrm{H}, \mathrm{I}, \mathrm{L}, \mathrm{S}$ & --- & A \\
\hline Jatropha curcas & $\mathrm{D}, \mathrm{F}, \mathrm{P}, \mathrm{X}$ & $\mathrm{V}$ & --- \\
\hline Jatropha gossypiifolia & $\mathrm{D}, \mathrm{I}, \mathrm{S}, \mathrm{X}$ & $\begin{array}{c}--- \\
-\cdots\end{array}$ & $\mathrm{Y}$ \\
\hline Lippia alba & C, F, I, W & G & --- \\
\hline Lippia micromera & $\mathrm{C}, \mathrm{G}, \mathrm{I}, \mathrm{L}, \mathrm{W}$ & $\mathrm{K}$ & ---- \\
\hline Melochia villosa & A, G, L & $\mathrm{L}$ & --- \\
\hline Momordica charantia & $\mathrm{A}, \mathrm{C}, \mathrm{H}, \mathrm{J}, \mathrm{M}, \mathrm{T}$ & $\mathrm{I}, \mathrm{L}, \mathrm{S}$ & ---- \\
\hline Mucuna urens & $\mathrm{A}, \mathrm{B}, \mathrm{S}$ & G & --- \\
\hline Neurolaena lobata & F, H, M, P, S & ---- & $\mathrm{K}$ \\
\hline Ocimum micranthum & $\mathrm{A}, \mathrm{F}$ & $\mathrm{G}, \mathrm{I}, \mathrm{R}$ & $\mathrm{H}, \mathrm{L}$ \\
\hline Odontadenia puncticulosa & B & ---- & $\mathrm{F}$ \\
\hline Pachira aquatica & $\mathrm{D}, \mathrm{S}, \mathrm{T}$ & $\mathrm{S}$ & I \\
\hline Passiflora quadrangularis & $\mathrm{A}, \mathrm{F}$ & $\mathrm{J}, \mathrm{P}$ & $\mathrm{M}, \mathrm{S}$ \\
\hline Pentaclethra macroloba & $\mathrm{B}, \mathrm{F}, \mathrm{S}$ & ---- & $\mathrm{L}$ \\
\hline Peperomia pellucida & $\mathrm{B}, \mathrm{I}, \mathrm{V}$ & ---- & $\mathrm{W}$ \\
\hline Peperomia peltata & $\mathrm{B}, \mathrm{I}, \mathrm{V}$ & $\mathrm{W}$ & ---- \\
\hline Persea americana & $\mathrm{D}, \mathrm{L}, 0, \mathrm{~W}$ & --- & $\mathrm{J}$ \\
\hline Petiveria alliacea & A, D & $\mathrm{R}$ & $\mathrm{L}, \mathrm{O}$ \\
\hline Piper auritum & $\mathrm{A}, \mathrm{C}, \mathrm{F}, \mathrm{G}$ & $\mathrm{L}$ & ---- \\
\hline Piper peltatum & A, F, G & ---- & $\mathrm{C}$ \\
\hline Psidium guajava & $\mathrm{D}, \mathrm{G}, \mathrm{H}, \mathrm{I}, \mathrm{S}$ & $\mathrm{P}$ & $\mathrm{F}$ \\
\hline Saccharum officinarum & $\mathrm{D}, \mathrm{I}, \mathrm{L}$ & ---- & $\mathrm{S}$ \\
\hline Sida acuta & $\mathrm{A}, \mathrm{C}, \mathrm{W}$ & $\mathrm{L}$ & ---- \\
\hline Spondias purpurea & $\mathrm{D}, \mathrm{F}$ & $\mathrm{S}$ & --- \\
\hline Stachytarpheta cayennensis & G, P, X & ---- & $\mathrm{F}$ \\
\hline Stachytarpheta jamaicensis & F, P, X & ---- & $\mathrm{G}$ \\
\hline Struthanthus cassythoides & A, B, S & $\mathrm{L}$ & --- \\
\hline Tagetes erecta & A, L, W & ---- & G \\
\hline Wedelia trilobata & $\mathrm{B}, \mathrm{F}, \mathrm{L}, \mathrm{W}$ & I & --- \\
\hline Zingiber officinale & $\mathrm{G}, \mathrm{L}$ & ---- & $\mathbf{F}$ \\
\hline
\end{tabular}




\section{Bibliografía}

Barrett, B. 1994. Medicinal plants of Nicaragua's Atlantic Coast. Economic Botany 48:8-20.

Bell, C. N. 1989. Tangweera: life and adventures among gentle savages. University of Texas Press, Austin, TX. (Originally published in 1899).

Boom, B. M. 1987. Ethnobotany of the Chacobo Indians, Beni, Bolivia. Advances in Economic Botany 4:1-68.

Cambie, R. C., and J. E. Ash. 1994. Fijian medicinal plants. CSIRO (Commonwealth Scientific and Industrial Research Organisation), Australia.

CIDCA (Centro de Investigaci6n y Documentacion de la Costa Atlantica). 1982. Demografia Costenia. CIDCA, Managua, Nicaragua. . 1985. Gramdtica Miskita. MIDNIRA, Managua, Nicaragua. . 1986. Diccionario Elemental: Miskito-Español-Español-Miskito. Editado e impreso en MIDNIRA, Managua, Nicaragua. . 1989. Diccionario elemental del Sumu: Sumu Meridional. Centro de Ciencia Cognitiva, Instituto Tecnol6gicod e Massachusetts, Cambridge,M A.

Coe, F. G. 1994. Ethnobotanyo f the Garifunao f east-ern Nicaragua. Ph.D. dissertation, University of Connecticut, Storrs, CT., and G. J. Anderson. 1996a. Ethnobotanyo pf the Gariffuna of eastern Nicaragua. Economic Botany 50:71-107. , and . 1996b. Screening of medicinal plants used by the Garifuna of eastern Nicaragua. Journalo Ethnopharmacology53:29-50. , and . 1997. Ethnobotany of the Miskitu of eastern Nicaragua. Journal of Ethnobiology 17:171-214.

Conzemius, E. 1932. Ethnographical survey of the Miskito and Sumu Indians of Honduras and Nicaragua. Bureau of American Ethnology Bulletin No. 106. U.S. Government Printing Office, Washington, DC.

Cronquist, A. 1981. An integrated system of classification of flowering plants. Columbia University Press, New York, NY.

Dennis, P. A. 1988. Herbal medicine among the Miskito of eastern Nicaragua. Economic Botany 42: 16-28.

Dozier, C. L. 1985. Nicaragua's Moskito shore: the years of British and American presence. The University of Alabama Press, Tuscaloosa.

Duke, J. A. 1994. Chemical composition of Belizean plants discussed in rainforest remedies: one hundred healing herbs of Belize. New York Botanical Garden, Bronx, NY.

Fey, U., and M. Sindel. 1993. Plantas medicinales de los Sumus. Imprenta Universidad Centroamericana (UCA), Managua, Nicaragua.

Garcia-Barriga, H. 1992. Flora medicinal de Colombia. Tomo 1-III. Tercer Mundo Editores, Bogota, Colombia.

Green, T. 1997. Neologismos en el idioma Sumu (Sumu Meridional). Wani 21:41-43.

Guerrero, J. N., and L. Soriano de Guerrero. 1985. Diccionario Nicaragüense: geografico e histórico. Editorial Somarriba, Masaya,Nicaragua.

Hale, K. 1991. El Ulwa (Sumu Meridional): un idioma distinto? Wani 11:27-50. 
Hale, C., and E. T. Gordon. 1987. Costefno demography: historical and contemporary demography of Nicaragua's Atlantic Coast. Pages 7-31 in CIDCA, ed., Ethnic groups and the nation state: the case of the Atlantic Coast in Nicaragua. University of Stockholm, Stockholm, Sweden.

Hegnauer, R. 1962-1994. Chemotaxonomie der Pflanzen. Vol. 1-1 la. Birkhauser Verlag, Basel, Switzerland.

Incer, J. 1975. Geograffa ilustrada de Nicaragua. Editora y Distribuidora, Nicaragtiense, S.A., Managua, Nicaragua. .1985. Toponomías indígenas de Nicaragua. Libro Libre, San Jose, Costa Rica.

McLean, M. E. 1996. Diccionario Panamahka. CID-CA, Managua, Nicaragua.

Morton, J. F. 1981. Atlas of medicinal plants of Middle America. Charles C. Thomas Publisher, Springfield, IL. . 1987. Fruits of warm climates. Media Incorporated, Greensboro, NC.

Nietschmann, B. Q. 1969. The distribution of Miskito, Sumu, and Rama Indians, Eastern Nicaragua. Bul-letin of the International Comittee on Urgent Anthropological and Ethnological Research 11:91- 102. 1979.Caribbeane dge: the coming of modern times to isolated people and wildlife. Bobbs Merrill, New York.

Norwood, S. 1997. Gramatica de la lengua Sumu. CIDCA-UCA, Managua, Nicaragua.

Roberts, 0. W. 1827. Narrative of voyages and excursions on the East Coast and the Interior of Central America. 1965 reprint, a facsimile of the 1827 edition. University of Florida Press, Gainesville, FL.

Romero, G. 1995. Las sociedades del atlántico de Nicaragua en los siglos XVII y XVIII. Banco Nicaragiiense, Managua, Nicaragua. F. de Oro Solorzano, M. Rizo, M. Membreño, A. Castegnaro de Foletti, J. Aviles, and B. Munioz. 1992. Persistencia indigena en Nicaragua. CIDCA, Managua, Nicaragua.

Salter, E. A. 1950. Plantas económicas y ornamentales de Nicaragua. Imprenta La Salle, Bluefields, Nicaragua. Schultes, R. E., and F. Raffauf. 1990. The healing forest medicinal and toxic plants of the Northwest Amazonia. Dioscorides Press, Portland, OR.

Seymour, F. C. 1980. A checklist of the vascular plants of Nicaragua. Phytologia Memoirs I:iii-x, 1-314.

Smutko, G. 1985. La Mosquitia: historia y cultura de la Costa Atlántica. Editorial La Ocarina, Managua, Nicaragua.

Taylor, B. W. 1962. The status and development of the Nicaraguan pine savannas. Caribbean Forester 23:21-26. 1963. An outline of the vegetation of Nicaragua. Journal of Ecology 51:27-54.

Tyler, V. E., L. R. Brady, and J. E. Robbers. 1985. Pharmacognosy. Lea and Febiger, Philadelphia, PA. Willaman, J. J., and Hui-Lin Li. 1970. Alkaloid-bearing plants and their contained alkaloids. Lloydia 33:1-286., and B. G. Schubert. 1961. Alkaloid-bearing plants and their contained alkaloids. U.S. Depart-ment of Agriculture, Technical Bulletin No. 1234, Washington, DC.

Williamson, D., J. Aviles, and M. McLean. 1993. Aspectos Generales de las Comunidades Sumus de la RAAN. Wani $14: 18-27$. 


\section{APÉNDICE}

\section{Listado de Plantas Usadas por los Ulwas'}

\begin{tabular}{|c|c|c|c|c|c|c|c|c|}
\hline Nombre Cientifico' & Nombre Comun² & Usos $^{3}$ & $\begin{array}{l}\text { Aplicaciones } \\
\text { Medicinales }\end{array}$ & $\begin{array}{l}\text { Materiales } \\
\text { Usados }^{5}\end{array}$ & $\begin{array}{c}\text { Modo de } \\
\text { Preparacion }\end{array}$ & $\begin{array}{l}\text { Modo de } \\
\text { Administra- } \\
\text { cion? }\end{array}$ & $\begin{array}{l}\text { Presencia } \\
\text { Alcaloide/ } \\
\text { glucosido }\end{array}$ & \# Etiqueta ${ }^{9}$ \\
\hline \multicolumn{9}{|l|}{ MICROPHYLLOPHYTA } \\
\hline \multicolumn{9}{|l|}{ GLOSSOPSIDA } \\
\hline \multicolumn{9}{|l|}{ SELAGINELLACEAE } \\
\hline 1. Selaginella sertata Spring & wî sangka (u) & M & $\mathrm{F}, \mathrm{I}$ & $\mathrm{P}$ & D & 0 & + & 4357 \\
\hline \multicolumn{9}{|l|}{ PTERIDOPHYTA } \\
\hline \multicolumn{9}{|l|}{ FILICOPSIDA } \\
\hline \multicolumn{9}{|l|}{ ADIANTHACEAE } \\
\hline 2. Acrostichum aureum L. & nawah damaska $(\mathrm{u})$ & $\mathrm{M}, \mathrm{O}$ & $F, Y$ & $L, R$ & D & 0 & $+/+L^{\mathrm{a}}$ & 4435 \\
\hline \multicolumn{9}{|l|}{ POLYPODIACEAE } \\
\hline $\begin{array}{l}\text { 3. Pityrogramma calomelanos } \\
\text { (L.) Link }\end{array}$ & wî pihka (u) & $\mathrm{M}, \mathrm{O}$ & $\mathrm{F}$ & $\mathrm{L}$ & D & $\mathrm{O}$ & $\mathrm{O} / \mathrm{O}$ & 4058 \\
\hline \multicolumn{9}{|l|}{ CONIFEROPHYTA } \\
\hline \multicolumn{9}{|l|}{ CONIFEROPSIDA } \\
\hline \multicolumn{9}{|l|}{ PINACEAE } \\
\hline 4. Pinus caribaea Morelet & wâlang panka (u) & $\mathrm{M}, \mathrm{O}$ & $A, L$ & S & $P$ & $\mathrm{I}, \mathrm{T}$ & $\mathrm{N} / \mathrm{O}$ & 4430 \\
\hline \multicolumn{9}{|l|}{ MAGNOLIOPHYTA } \\
\hline \multicolumn{9}{|l|}{ MAGNOLIOPSIDA ( DICOTS) } \\
\hline \multicolumn{9}{|l|}{ ACANTHACEAE } \\
\hline 5. Blechum brownei Juss. & kunsil (u) & M & $B, D$ & $L, P$ & D & 0 & + & 3631 \\
\hline 6. Justicia spicigera Schlecht. * & $\begin{array}{l}\text { damaska sangka } \\
\text { (u) }\end{array}$ & $\mathrm{M}, \mathrm{O}$ & $\mathrm{F}$ & L & D & 0 & $\mathrm{O} /+\mathrm{L}^{\mathrm{d}}$ & 4463 \\
\hline
\end{tabular}

${ }^{1}$ Para la completa comprensión y el uso correcto de la presente tabla que contiene la información sobre las plantas usadas por los ulwas, hemos elaborado las siguientes notas:

${ }^{1}$ Nombre científico de las familias de angiospermas que sigue Cronquist (1981), las dicotiledóneas y monocotiledóneas, sus familias, géneros y especies se encuentran en orden alfabético.

${ }^{2}$ Nombres Comunes: $\mathrm{c}=$ Ingles Criollo; $\mathrm{s}=$ Español; $\mathrm{m}$ = Miskitu; $\mathrm{u}=$ Ulwa; la ortografía se basa en CIDCA $(1985,1986,1989)$ y Smutko (1985). De acuerdo con CIDCA (1989) el alfabeto de los Ulwa consiste de las siguientes letras: $\underline{\mathbf{a}}, \hat{\mathbf{a}}, \mathbf{b}, \mathbf{d}, \mathbf{g}, \mathbf{h}, \mathbf{i}, \hat{\mathbf{1}}, \mathbf{k}, \mathbf{l}, \mathbf{m}, \mathbf{n}, \mathbf{p}, \mathbf{r}, \mathbf{s}, \mathbf{t}, \mathbf{u}, \hat{\mathbf{u}}, \mathbf{w}, \mathbf{y}$. Las vocales con signos diacríticos se pronuncian a larga duración, e.g., sûtak es pronunciada "suutak," wî ies pronunciada "wii," y yâmak es pronunciada "yaamak."

${ }^{3}$ Usos: $\mathrm{F}=$ Comida; $\mathrm{M}=$ Medicinal; $\mathrm{O}$ = otros (construcción, artesanías, fibras, colorantes)

${ }^{4}$ Aplicaciones medicinales: $\mathrm{A}=$ Dolores y molestias; $\mathrm{B}=$ mordidas y picaduras (serpiente, escorpión, insectos); $\mathrm{C}=$ Embarazo y parto; $\mathrm{D}=\mathrm{Diarrea} ; \mathrm{E}=\mathrm{Emético}$;

F = Fiebre; G = Digestivo; (dolores de estomago, ulceras, etc.); H = Hipertensión; I = Infecciones; J = Diabetes; K = Diurético; L = Problemas Respiratorios y Pulmonares (gripa, tos, etc.); $\mathrm{M}=$ Malaria;

$\mathrm{N}$ = Quemaduras; $\mathrm{O}=$ Abortivos; $\mathrm{P}=$ Lombrices y parásitos intestinales; $\mathrm{Q}=$ Astringente; $\mathrm{R}=$ Rituales; $\mathrm{S}=$ Erupciones de la piel y llagas; $\mathrm{T}=$ Tónicos y Anemia (fortificantes de sangre); $\mathrm{U}=$ Cortaduras y hemorragias; $\mathrm{V}=$ Enfermedades Venéreas; $\mathrm{W}=$ Molestias femeninas (Menstruación, Hemorragia); $\mathrm{X}=\mathrm{Purgante}$ y Laxante; $\mathrm{Y}=$ Constipación; $\mathrm{Z}=$ Extracción de dientes

${ }^{5}$ Materiales Usados: $\mathrm{B}=$ Corteza $\mathrm{C}=$ Flor $\mathrm{E}=$ Semillas, $\mathrm{F}=$ Fruta $\mathrm{L}=$ Hoja $\mathrm{M}=$ Tallo; $\mathrm{P}=$ Toda la planta $; \mathrm{R}=$ Raíz; $\mathrm{S}=$ Savia.

${ }^{6}$ Modo of Preparación: (Ver sección en medicinales para una explicación más a fondo.) B = Baño; D = Decocción; I = Infusión; J = Jugo extraido de las partes; N = Ninguno; $\mathrm{P}=$ Cataplasma; $\mathrm{S}=$ Jarabe.

${ }^{7}$ Modo of Administración: (Ver sección en medicinales para una explicación más a fondo). B = Baño; $\mathrm{I}=$ Inhalación; $\mathrm{O}=$ Oral; $\mathrm{T}=$ Tópica.

${ }^{8}$ Prueba de Alcaloides/Glucósidos. Prueba Alcaloide: $\mathrm{N}=$ no prueba no búsqueda de literatura; $-\mathrm{L}=$ no literatura; $+\mathrm{L}=$ Alcaloides reportados en la literatura; + (presente)/ $\mathrm{O}$ (ausente) en la prueba de Coe (Ver Métodos y Materiales). Prueba de Glucósidos: La búsqueda de literatura acerca de glucósidos fue limitada y solo se llevo a cabo con las especies que resultaron negativas en la prueba de alcaloides; $/+\mathrm{L}=$ presente, $/ \mathrm{O}=$ no reportado.

${ }^{9}$ Numero de Etiqueta: $\mathrm{C}=$ Introducida común y/o naturalizada, una o no wtiqueta recolectada; $\mathrm{N}=$ Nativa común, solo una etiqueta recolectada por todos los grupos; $\mathrm{NV}$ $=$ No Etiqueta; $\mathrm{P}=$ Compradas en mercados

regionales y tiendas en ciudades más grandes, las cuales no crecen en el oriente de Nicaragua; \# = F.G. Coe números de acceso

a=Cambie and Ash 1994; b=Duke 1994; c=García-Barriga 1992; d=Hegnauer 1962-1994; e=Morton 1981, 1987; f=Tyler, Brady, and Robbers 1985; g=Willaman and Hui-Lin Li 1970; h=Willaman and Shubert 1961

*=Species únicas de los Ulwa. 
WANI

\begin{tabular}{|c|c|c|c|c|c|c|c|c|}
\hline Nombre Gientifico ${ }^{1}$ & Nombre Gomun² & Usos $^{3}$ & $\begin{array}{l}\text { Aplicaciones } \\
\text { Medicinales }\end{array}$ & $\begin{array}{c}\text { Materiales } \\
\text { Usados }^{5}\end{array}$ & $\begin{array}{c}\text { Modo de } \\
\text { Preparacion }\end{array}$ & $\begin{array}{l}\text { Modo de } \\
\text { Administra- } \\
\text { cion? }\end{array}$ & $\begin{array}{l}\text { Presencia } \\
\text { Alcaloide/ } \\
\text { glucosido }\end{array}$ & \# Etiqueta9 \\
\hline \multicolumn{9}{|l|}{ ANACARDIACEAE } \\
\hline 7. Anacardium occidentale L. & kasauh (u) & $\mathrm{F}, \mathrm{M}, \mathrm{O}$ & $A, D, F, S$ & B & D & $0, T$ & + & 2725 \\
\hline 8. Mangifera indica $\mathrm{L}$. & mankru, yalau (u) & $\mathrm{F}, \mathrm{M}, \mathrm{O}$ & $A, D, S$ & $\mathrm{~B}, \mathrm{~L}$ & $\mathrm{D}$ & $0, T$ & + & 3387 \\
\hline 9. Spondias mombin L. & walak (u) & $\mathrm{F}, \mathrm{M}, \mathrm{O}$ & $D, F, I, S$ & $\mathrm{~B}, \mathrm{~L}$ & $\mathrm{D}$ & 0 & $\mathrm{O} / \mathrm{O}$ & 2275 \\
\hline 10._S. purpurea L. & walak (u) & $\mathrm{F}, \mathrm{M}, \mathrm{O}$ & $D, F$ & $\mathrm{~B}, \mathrm{~L}$ & $\mathrm{D}$ & 0 & $\mathrm{O} / \mathrm{O}$ & 2959 \\
\hline \multicolumn{9}{|l|}{ ANNONACEAE } \\
\hline 11. Annona glabra $\mathrm{L}$. & saput (u) & $F, M$ & $A, C$ & $\mathrm{~B}, \mathrm{~L}$ & $D, P$ & $0, T$ & $+\mathrm{L}^{\mathrm{h}}$ & 2403 \\
\hline 12. A. muricata L. & saput (u) & $F, M$ & $C, D$ & $\mathrm{~B}, \mathrm{~L}$ & $\mathrm{D}$ & 0 & $+\mathrm{L}^{\mathrm{h}}$ & 3392 \\
\hline $\begin{array}{l}\text { 13. Guatteria amplifolia Triana } \\
\text { \& Planch. }\end{array}$ & tastala $(\mathrm{u})$ & $M$ & $D, V$ & $\mathrm{~B}, \mathrm{~L}$ & $\mathrm{D}$ & 0 & + & 2429 \\
\hline \multicolumn{9}{|l|}{ APIACEAE } \\
\hline 14. Eryngium foetidum $\mathrm{L}$. & kisauri (u) & $F, M$ & $D, G, P, R$ & $\mathrm{~L}$ & $D, I$ & $\mathrm{~B}, \mathrm{O}$ & $\mathrm{O} /+\mathrm{L}^{\mathrm{c}}$ & 3515 \\
\hline \multicolumn{9}{|l|}{ APOCYNACEAE } \\
\hline 15. Allamanda cathartica L. & tumi lalahka (u) & $\mathrm{M}, \mathrm{O}$ & $E, X$ & $F, L, S$ & $\mathrm{D}$ & 0 & $+/+L^{d}$ & 2522 \\
\hline 16. Odontadenia puncticulosa & sakaluk (u) & $M$ & $B, F$ & $\mathrm{~L}$ & D & 0 & + & 2139 \\
\hline \multicolumn{9}{|l|}{ (Rich.) Pull. } \\
\hline $\begin{array}{l}\text { 17.Tabernaemontana chryso- } \\
\text { carpa Blake }\end{array}$ & waku (u) & M & $\mathrm{F}, \mathrm{I}$ & $\mathrm{L}, \mathrm{S}$ & $\mathrm{D}$ & 0 & + & 4193 \\
\hline \multicolumn{9}{|l|}{ ARISTOLOCHIACEAE } \\
\hline 18. Aristolochia trilobata L. & kuntribu (u) & $\mathrm{M}, \mathrm{O}$ & $\mathrm{B}, \mathrm{G}, \mathrm{L}, \mathrm{T}$ & $L, P$ & $\mathrm{D}, \mathrm{I}$ & 0 &,$+ L^{\mathrm{d}}$ & 3923 \\
\hline \multicolumn{9}{|l|}{ ASCLEPIADACEAE } \\
\hline 19. Asclepias curassavica L. & wahbara (u) & M & $\mathrm{B}, \mathrm{F}, \mathrm{P}, \mathrm{S}$ & $\mathrm{L}$ & $D, P$ & $\mathrm{O}, \mathrm{T}$ &,$++L^{h}$ & 3235 \\
\hline \multicolumn{9}{|l|}{ ASTERACEAE } \\
\hline 20. Bidens riparia H.B.K. & uhdanaka dî basta (u) & M & $\mathrm{L}$ & L & D & 0 & + & 3225 \\
\hline 21. Elephantopus mollis H.B.K. & slimsi (u) & M & $D, P$ & $\mathrm{~L}$ & $\mathrm{D}$ & 0 & + & 3354 \\
\hline 22. E. spicatus (Juss.) C. F. Baker & damaska pamkih (u) & $M$ & A & $\mathrm{L}$ & $\mathrm{J}$ & $\mathrm{T}$ & + & 4470 \\
\hline $\begin{array}{l}\text { 23. Eleutheranthera ruderalis } \\
\text { (Sw.) Schltdl. }\end{array}$ & walalaka (u) & M & $F, S$ & $\mathrm{~L}$ & $\mathrm{D}$ & $0, T$ & + & 2219 \\
\hline $\begin{array}{l}\text { 24. Lasianthaea fruticosa (L.) } \\
\text { M. Becker * }\end{array}$ & pulu lalahka (u) & $M$ & $\mathrm{~L}$ & $\mathrm{~F}$ & D & 0 & + & \\
\hline 25. Matricaria chamomilla L. & wâlang (u) & $M$ & G & $P$ & $\mathrm{D}$ & 0 & $+L^{\mathrm{g}}$ & 4434 \\
\hline $\begin{array}{l}\text { 26. Mikania cordifolia_(L.f.) } \\
\text { Willd. }\end{array}$ & kunsisil (u) & M & $A, B, F$ & $L, M, P$ & $D, P$ & $0, T$ &,$++L^{h}$ & 3312 \\
\hline 27. Neurolaena lobata (L.) R. Br & kunata palska (u) & $M$ & $F, H, K, M, P, S$ & $\mathrm{~L}$ & $\mathrm{D}$ & $0, T$ & $+L^{d}$ & 2548 \\
\hline 28. Tagetes erecta $\mathrm{L}$. & baram (u) & M & $\mathrm{A}, \mathrm{F}, \mathrm{G}, \mathrm{L}, \mathrm{W}$ & L,P & D & 0 & $+L^{\mathrm{g}}$ & 2762 \\
\hline $\begin{array}{l}\text { 29. Vernonia scorpioides (Lam.) } \\
\text { Pers. * }\end{array}$ & malakasa (u) & $M$ & $R, T$ & $L, R$ & $B, D$ & $\mathrm{~B}, \mathrm{O}$ & $\mathrm{O} / \mathrm{O}$ & 4347 \\
\hline 30. Wedelia trilobata (L.) Hitchc. & mululuh (u) & $M$ & $B, F, L, W$ & $F, L, M$ & $\mathrm{D}$ & 0 & $\mathrm{O} / \mathrm{O}$ & 3556 \\
\hline \multicolumn{9}{|l|}{ BIGNONIACEAE } \\
\hline $\begin{array}{l}\text { 31. Arrabidaea chica (Humb., \& } \\
\text { Bonpl.) Verl. }\end{array}$ & damaska âwas (u) & $M$ & $\mathrm{D}$ & $\mathrm{L}$ & $\mathrm{D}$ & 0 & $+L^{c}$ & 4464 \\
\hline 32. Crescentia cujete L. & sutak, sûtak (u) & $M$ & $\mathrm{D}, \mathrm{F}, \mathrm{L}$ & $\mathrm{F}$ & $D, S$ & 0 & $\mathrm{O} /+\mathrm{L}^{\mathrm{c}}$ & 3447 \\
\hline \multicolumn{9}{|l|}{ BIXACEAE } \\
\hline 33. Bixa orellana $\mathrm{L}$. & awal (u) & $\mathrm{F}, \mathrm{M}, \mathrm{O}$ & $\mathrm{D}, \mathrm{L}, \mathrm{N}$ & $\mathrm{E}, \mathrm{L}$ & $D, I$ & $0, T$ & $+L^{b}$ & 3267 \\
\hline \multicolumn{9}{|l|}{ BOMBACACEAE } \\
\hline 34._Ceiba pentandra (L.) Gaertn. & panya, paniki (u) & $\mathrm{M}, \mathrm{O}$ & $A, E, K, Q$ & B & $\mathrm{D}$ & 0 & $\mathrm{O} / \mathrm{O}$ & 4445 \\
\hline 35. Pachira aquatica L. & pankasna $(\mathrm{u})$ & $\mathrm{M}, \mathrm{O}$ & $\mathrm{D}, \mathrm{I}, \mathrm{T}$ & $\mathrm{B}, \mathrm{E}$ & D & 0 & $\mathrm{O} / \mathrm{O}$ & 3384 \\
\hline
\end{tabular}




\begin{tabular}{|c|c|c|c|c|c|c|c|c|}
\hline Nombre Gientifico ${ }^{1}$ & Nombre Gomunn ${ }^{2}$ & Usos $^{3}$ & $\begin{array}{l}\text { Aplicaciones } \\
\text { Medicinales }\end{array}$ & $\begin{array}{l}\text { Materiales } \\
\text { Usados }^{5}\end{array}$ & $\begin{array}{c}\text { Modo de } \\
\text { Preparacion }\end{array}$ & $\begin{array}{c}\text { Modo de } \\
\text { Administra- } \\
\text { cion? }\end{array}$ & $\begin{array}{l}\text { Presencia } \\
\text { Alcaloide/ } \\
\text { glucosido }\end{array}$ & \# Etiqueta $^{9}$ \\
\hline \multicolumn{9}{|l|}{ BORAGINACEAE } \\
\hline $\begin{array}{l}\text { 36. Cordia alliodora (Ruiz \& } \\
\text { Pav.) Oken }\end{array}$ & wingkurh (u) & $\mathrm{M}, \mathrm{O}$ & $\mathrm{S}, \mathrm{T}$ & L & D & $0, T$ & $-\mathrm{L} / \mathrm{O}$ & 4366 \\
\hline $\begin{array}{l}\text { 37. Cordia curassavica (Jacq.) } \\
\text { Roem. \& Schult. }\end{array}$ & kuyus (u) & $\mathrm{F}, \mathrm{M}$ & $A, C, D, F, H$ & L & D & $\mathrm{B}, \mathrm{O}, \mathrm{T}$ & + & 3653 \\
\hline 38. Cordia spinescens $\mathrm{L}$. & kaya (u) & M & $A, C, F, H$ & $\mathrm{~L}$ & $\mathrm{D}$ & 0 & + & 3875 \\
\hline 39. Heliotropium indicum L. & wâkurus umah (u) & $M$ & $B, D, S$ & $L, P$ & $\mathrm{D}$ & 0 & $+L^{h}$ & 4018 \\
\hline \multicolumn{9}{|l|}{ BURSERACEAE } \\
\hline 40. Bursera simaruba (L.) Sarg. & limsi (m) & $\mathrm{M}, \mathrm{O}$ & $\mathrm{I}, \mathrm{S}, \mathrm{T}$ & $B$ & $\mathrm{D}$ & $\mathrm{B}, \mathrm{O}$ & $\mathrm{O} / \mathrm{O}$ & 3824 \\
\hline $\begin{array}{l}\text { 41. Tetragastris panamensis } \\
\text { (Engl.) Kuntze }\end{array}$ & sakal (u) & 0 & - & - & - & - & - & 4383 \\
\hline \multicolumn{9}{|l|}{ CARICACEAE } \\
\hline 42. Carica papaya L. & ulmak, ulumak (u) & $\mathrm{F}, \mathrm{M}$ & $P, S, U, Y$ & $F, L, S$ & $D, J$ & $0, T$ & $+L^{\mathrm{g}}$ & 2723 \\
\hline \multicolumn{9}{|l|}{ CECROPIACEAE } \\
\hline 43. Cecropia peltata_L. & palang (u) & M & W & L & D & 0 & $+\mathrm{L}^{\mathrm{e}}$ & 3462 \\
\hline \multicolumn{9}{|l|}{ CHRYSOBALANACEAE } \\
\hline 44. Chrysobalanus icaco L. & tawa $(u)$ & $F, M$ & $D, Q$ & $B, R$ & $\mathrm{D}$ & 0 & $\mathrm{O} / \mathrm{O}$ & 2838 \\
\hline 45. C. pellocarpus G. Mey. & baraska siuli $(u)$ & $\mathrm{F}, \mathrm{M}$ & $D, Q$ & $B, R$ & $\mathrm{D}$ & 0 & $\mathrm{O} / \mathrm{O}$ & 2136 \\
\hline \multicolumn{9}{|l|}{ CLUSIACEAE } \\
\hline $\begin{array}{l}\text { 46._Calophyllum brasiliense } \\
\text { Cambess }\end{array}$ & awanak (u) & $\mathrm{M}, \mathrm{O}$ & $A, L$ & $\mathrm{~B}, \mathrm{~S}$ & D & O,T & $-\mathrm{L} / \mathrm{O}$ & 4371 \\
\hline 47. Garcinia mangostana L. & mangosteen (c) & $\mathrm{F}$ & A & $\mathrm{B}, \mathrm{S}$ & $\mathrm{N}, \mathrm{P}$ & $T$ & $+L^{e}$ & NV \\
\hline 48. Symphonia globulifera_L.f. & paumaba (u) & 0 & - & - & - & - & - & 2356 \\
\hline \multicolumn{9}{|l|}{ COMBRETACEAE } \\
\hline 49. Conocarpus erectus L. & mankru (u) & $\mathrm{M}, \mathrm{O}$ & $\mathrm{D}, \mathrm{Q}$ & $B, L$ & $\mathrm{D}$ & 0 & $\mathrm{O} / \mathrm{O}$ & 2775 \\
\hline 50. Laguncularia racemosa_(L.) & mankru pihka (u) & $\mathrm{M}, \mathrm{O}$ & $\mathrm{D}$ & B & D & 0 & $\mathrm{O} / \mathrm{O}$ & 2712 \\
\hline \multicolumn{9}{|l|}{ Gaertn. } \\
\hline $\begin{array}{l}\text { 51. Terminalia amazonica (J. F. } \\
\text { Gmel.) Exell * }\end{array}$ & $\operatorname{limnah}(u)$ & $\mathrm{M}, \mathrm{O}$ & D & B & D & 0 & $\mathrm{O} / \mathrm{O}$ & 4465 \\
\hline 52. T. catappa L. & amans, îbu (u) & $\mathrm{F}, \mathrm{O}$ & - & - & - & - & - & 2708 \\
\hline $\begin{array}{l}\text { 53. T. oblonga (Ruiz \& Pav. ) } \\
\text { Steud. }\end{array}$ & limnah (u) & $\mathrm{O}$ & - & - & - & - & - & 4388 \\
\hline \multicolumn{9}{|l|}{ CONNARACEAE } \\
\hline $\begin{array}{l}\text { 54. Connarus lambertii (DC.) } \\
\text { Sagot }\end{array}$ & siuli pauka (u) & $\mathrm{M}, \mathrm{O}$ & $Q$ & $B, L$ & D & 0 & + & 2124 \\
\hline \multicolumn{9}{|l|}{ CONVOLVULACEAE } \\
\hline 55. Cuscuta americana_L. & $\begin{array}{ll}\text { asang } & \text { wahka } \\
\text { lalahka (u) } & \end{array}$ & $M$ & $\mathrm{~S}$ & L,M & $D, P$ & $T$ & + & 2129 \\
\hline 56. Ipomoea batatas (L.) Lam. & pai (u) & $F, M$ & $U$ & $\mathrm{~L}$ & D & $T$ & + & 3637 \\
\hline 57. I. mauritiana Jacq. & salalani (u) & $M$ & $\mathrm{~B}, \mathrm{~S}$ & $\mathrm{~L}$ & $D, P$ & $0, T$ & $\mathrm{O} / \mathrm{O}$ & 4318 \\
\hline 58. I. pes-caprae (L.) R.Br. & $\begin{array}{l}\text { pulu kuma kungka } \\
\text { (u) }\end{array}$ & $M$ & $\mathrm{~F}, \mathrm{~S}, \mathrm{~T}$ & L & $D, P$ & O,T &,$++L^{g}$ & 2003 \\
\hline 59. I. setifera Poir * & tutuk, ulupuy (u) & $M$ & $\mathrm{~B}, \mathrm{~S}$ & L & $D, P$ & $0, T$ & + & 3371 \\
\hline $\begin{array}{l}\text { 60. Operculina pteripes (G. Don) } \\
\text { O'Donell }\end{array}$ & bitta tataku $(\mathrm{m})$ & M & $B, U$ & $L$ & $P$ & $T$ & + & 4102 \\
\hline \multicolumn{9}{|l|}{ CRASSULACEAE } \\
\hline $\begin{array}{l}\text { 61. Kalanchoe pinnata (Lam.) } \\
\text { Pers. }\end{array}$ & pan sangka (u) & $M$ & $A, L$ & $\mathrm{~L}$ & $D, P$ & $0, T$ & $\mathrm{O} /+\mathrm{L}^{\mathrm{b}}$ & 3432 \\
\hline
\end{tabular}


WANI

\begin{tabular}{|c|c|c|c|c|c|c|c|c|}
\hline Nombre Cientifico' & Nombre Comun² & Usos $^{3}$ & $\begin{array}{l}\text { Aplicaciones } \\
\text { Medicinales }\end{array}$ & $\begin{array}{l}\text { Materiales } \\
\text { Usados }^{5}\end{array}$ & $\begin{array}{c}\text { Modo de } \\
\text { Preparacion' }\end{array}$ & $\begin{array}{l}\text { Modo de } \\
\text { Administra- } \\
\text { cion? }\end{array}$ & $\begin{array}{l}\text { Presencia } \\
\text { Alcaloide/ } \\
\text { glucosido }\end{array}$ & \# Etiqueta9 \\
\hline \multicolumn{9}{|l|}{ CUCURBITACEAE } \\
\hline 62. Fevillea cordifolia L. & mula (u) & M & $A, B, E, G$ & $\mathrm{E}$ & $\mathrm{I}, \mathrm{P}$ & $\mathrm{O}, \mathrm{T}$ & $\mathrm{O} / \mathrm{O}$ & 2765 \\
\hline 63. Momordica charantia L. & $\begin{array}{l}\text { panaminik, } \\
\text { makalalaska, } \\
\text { miniklalasni (u) }\end{array}$ & $F, M$ & $\mathrm{~A}, \mathrm{C}, \mathrm{H}, \mathrm{J}, \mathrm{M}, \mathrm{T}$ & $L, M$ & D & 0 &,$++L^{h}$ & 3635 \\
\hline \multicolumn{9}{|l|}{ DILLENIACEAE } \\
\hline 64. Davilla kunthii A. St. Hil. & wî babatka (u) & $\mathrm{M}, \mathrm{O}$ & $\mathrm{D}, \mathrm{Q}$ & $B, L, M$ & D & 0 & $+\mathrm{L}$ & 2705 \\
\hline \multicolumn{9}{|l|}{ EUPHORBIACEAE } \\
\hline $\begin{array}{l}\text { 65. Acalypha arvensis Poepp. } \\
\text { \& Endl. }\end{array}$ & kiskita (u) & M & $\mathrm{B}, \mathrm{S}$ & L,P & $\mathrm{D}$ & $0, T$ & $\mathrm{O} /+\mathrm{L}^{\mathrm{d}}$ & 3641 \\
\hline 66. Croton punctatus_L. & irik, kikis (u) & $M$ & $\mathrm{~F}, \mathrm{I}$ & $F, L, R$ & $\mathrm{D}$ & 0 & $+L^{\mathrm{g}}$ & 4046 \\
\hline $\begin{array}{l}\text { 67. Euphorbia thymifolia (L.) } \\
\text { Millsp. }\end{array}$ & baska, bisini (u) & M & $A, C, I$ & $\mathrm{~L}, \mathrm{P}$ & $\mathrm{D}$ & 0 & $+\mathrm{L}$ & 2224 \\
\hline $\begin{array}{l}\text { 68. Hyeronima alchorneoides } \\
\text { Allemão }\end{array}$ & nancitón (s) & 0 & - & - & - & - & - & 4364 \\
\hline 69. Jatropha curcas L. & pisik (u) & M & $D, F, P, X$ & $\mathrm{~L}, \mathrm{~S}$ & D & 0 & $+L^{h}$ & 2749 \\
\hline 70. J. gossypiifolia L. & kishka (u) & M & $D, I, S, X, Y$ & $\mathrm{~L}$ & $\mathrm{D}$ & 0 & $+L^{h}$ & 4344 \\
\hline 71. Manihot esculenta Crantz & malai, maley (u) & $\mathrm{F}, \mathrm{M}$ & $A, D, F$ & $L, R$ & $\mathrm{D}$ & 0 & $\mathrm{O} /+\mathrm{L}^{\mathrm{a}}$ & 3269 \\
\hline 72. Ricinus communis $\mathrm{L}$. & unapalan (u) & M & $A, F, X$ & $\mathrm{E}, \mathrm{L}$ & $D, P$ & $0, T$ & $+\mathrm{L}^{\mathrm{h}}$ & 3507 \\
\hline \multicolumn{9}{|l|}{ FABACEAE } \\
\hline 73. Abrus precatorius $\mathrm{L}$. & John Crowbead (c) & M & - & $\mathrm{F}, \mathrm{L}$ & $\mathrm{D}$ & 0 & $+L^{\mathrm{h}}$ & 4026 \\
\hline 74. Arachis hypogaea L. & pinda (c) & $\mathrm{F}$ & - & - & - & - & - & 2752 \\
\hline 75. Cassia alata L. tata, tatah (u) & daka, papaih, & M & $\mathrm{F}, \mathrm{I}, \mathrm{S}, \mathrm{T}, \mathrm{X}$ & $\mathrm{F}, \mathrm{L}$ & $B, D, J, P$ & $\mathrm{~B}, \mathrm{O}, \mathrm{T}$ &,$++L^{h}$ & 3202 \\
\hline 76. C. hirsuta L. & kusnini (u) & M & $F, W$ & $\mathrm{E}, \mathrm{L}$ & D & 0 & $+L^{c}$ & 3584 \\
\hline 77. C. occidentalis L. & singsingya (u) & $\mathrm{M}$ & $\mathrm{F}, \mathrm{G}, \mathrm{I}, \mathrm{L}$ & $L, P, R$ & $\mathrm{D}, \mathrm{J}$ & $0, T$ &,$++L^{g}$ & 3625 \\
\hline 78. C. reticulata Willd. & tislin (u) & $M$ & $A, B, I, S, W, X$ & $L, R$ & $\mathrm{D}$ & 0 & + & 2799 \\
\hline 79. Crotalaria retusa $\mathrm{L}$. & $\begin{array}{l}\text { nangtal damaska } \\
\text { (u) }\end{array}$ & M & $S, U, X$ & $\mathrm{~L}$ & $D, P$ & $0, T$ & $+\mathrm{L}^{\mathrm{h}}$ & 4226 \\
\hline 80. C. verrucosa L. & pula suyuka (u) & M & $\mathrm{S}, \mathrm{X}$ & $\mathrm{L}$ & D & $0, T$ & $+L^{h}$ & 3718 \\
\hline $\begin{array}{l}\text { 81. Dahlbergia brownei (Jacq.) } \\
\text { Urb. }\end{array}$ & rusul (m) & $\mathrm{M}, \mathrm{O}$ & $D, Q, S$ & $B, L, M$ & $\mathrm{D}$ & $0, T$ & O/O & 4082 \\
\hline 82. D. hypoleuca Pittier & rusul $(\mathrm{m})$ & 0 & -- & -- & -- & -- & -- & 4325 \\
\hline 83. D. tucurensis Donn. Sm. & rusul $(\mathrm{m})$ & 0 & -- & -- & -- & -- & -- & 4391 \\
\hline $\begin{array}{l}\text { 84. Desmodium adscendens } \\
\text { (Sw.) DC. }\end{array}$ & $\begin{array}{l}\text { danka dangpanak } \\
\text { (u) }\end{array}$ & M & $A, D, G, I, S$ & $L, P, R$ & $D, I$ & 0 & + & 4117 \\
\hline $\begin{array}{l}\text { 85. D. barbatum (L.) Benth. \& } \\
\text { Oerst. }\end{array}$ & \begin{tabular}{|l} 
basaka danka \\
Dangpanak (u)
\end{tabular} & M & $\mathrm{A}, \mathrm{I}, \mathrm{S}, \mathrm{V}$ & $L, R$ & D & 0 & $\mathrm{O} / \mathrm{O}$ & 3309 \\
\hline $\begin{array}{l}\text { 86. D. canum (J.F. Gmel.) Schinz } \\
\text { \& Thell. }\end{array}$ & yamah damaska (u) & M & $\mathrm{A}, \mathrm{I}, \mathrm{S}$ & $L, R$ & D & 0 & + & 3673 \\
\hline 87. D. triflorum_(L.) DC. & kumalata (u) & $\mathrm{M}$ & $A, F, I$ & $L, R$ & $\mathrm{D}$ & 0 & + & 2767 \\
\hline 88. Dioclea megacarpa Rolfe & maklala (u) & $\mathrm{M}, \mathrm{O}$ & $\mathrm{A}, \mathrm{S}$ & $\mathrm{L}$ & $D, P$ & $\mathrm{~T}$ & + & 3236 \\
\hline $\begin{array}{l}\text { 89. Dipteryx oleifera (Benth.) } \\
\text { Taub. }\end{array}$ & îbu (u) & $\mathrm{F}, \mathrm{M}, \mathrm{O}$ & $A, F, Q$ & $B, F, M$ & $\mathrm{D}$ & 0 & $+L^{d}$ & 2321 \\
\hline 90. Hymenaea courbaril L. & tipi (u) & $\mathrm{M}, \mathrm{O}$ & $A, D, F, L$ & $\mathrm{~B}, \mathrm{~S}$ & $D, P$ & $0, T$ & $0 / 0$ & 3417 \\
\hline 91. Mimosa pudica $\mathrm{L}$. & $\operatorname{amin}(\mathrm{u})$ & $\mathrm{M}$ & $A, G, P, W$ & $L, M, R$ & D & 0 &,$++\mathrm{L}^{\mathrm{h}}$ & 2252 \\
\hline 92. Mucuna urens DC. & wabala (u) & M & $A, B, S$ & $\mathrm{~s}$ & $D, P$ & $\mathrm{~T}$ & $+\mathrm{L}^{\mathrm{h}}$ & 2870 \\
\hline 93. Pentaclethra macroloba & tikbus damaska (u) & $\mathrm{M}, \mathrm{O}$ & $B, F, L, S$ & $\mathrm{~B}$ & D & $0, T$ & + & 2441 \\
\hline \multicolumn{9}{|l|}{ (Willd.) Kuntz } \\
\hline 94. Phaseolus vulgaris $\mathrm{L}$. & $\sin a k(u)$ & $\mathrm{F}$ & - & - & - & - & - & 2758 \\
\hline
\end{tabular}




\begin{tabular}{|c|c|c|c|c|c|c|c|c|}
\hline Nombre Cientifico' & Nombre Gomun² & Usos $^{3}$ & $\begin{array}{l}\text { Aplicaciones } \\
\text { Medicinales }\end{array}$ & $\begin{array}{c}\text { Materiales } \\
\text { Usados }^{5}\end{array}$ & $\begin{array}{c}\text { Modo de } \\
\text { Preparacion }^{6}\end{array}$ & $\begin{array}{l}\text { Modo de } \\
\text { Administra- } \\
\text { cion? }^{7}\end{array}$ & $\begin{array}{l}\text { Presencia } \\
\text { Alcaloide/ } \\
\text { glucosido }\end{array}$ & \# Etiqueta $^{9}$ \\
\hline $\begin{array}{l}\text { 95. Pithecolobium dulce (Roxb.) } \\
\text { Benth. }\end{array}$ & samutka (u) & $\mathrm{M}, \mathrm{O}$ & $\mathrm{D}, \mathrm{Q}$ & B & D & 0 & $+L^{h}$ & 3764 \\
\hline \multicolumn{9}{|l|}{ GENTIANACEAE } \\
\hline 96. Coutoubea spicata Aubl. & wîtang tusnaka (u) & M & $A, F$ & $\mathrm{~L}$ & $\mathrm{D}$ & 0 & $\mathrm{O} / \mathrm{O}$ & 2587 \\
\hline \multicolumn{9}{|l|}{ LAMIACEAE } \\
\hline 97. Hyptis capitata Jacq. & $\begin{array}{l}\text { aras kasnin nuhni } \\
\text { (u) }\end{array}$ & M & $\mathrm{G}, \mathrm{L}$ & $L, P$ & D & 0 & $\mathrm{O} / \mathrm{O}$ & 3558 \\
\hline 98. _H. verticillata Jacq. & wahiwin saika (u) & M & $\mathrm{A}, \mathrm{H}, \mathrm{I}, \mathrm{L}, \mathrm{S}$ & $L, R$ & $D, I, P$ & $0, T$ & + & 2667 \\
\hline 99. Ocimum micranthum Willd. & kuma sirpi (u) & $F, M$ & $A, F, H, L$ & $\mathrm{~L}$ & $D, I$ & $0, T$ & $\mathrm{O} / \mathrm{O}$ & 2229 \\
\hline \multicolumn{9}{|l|}{ LAURACEAE } \\
\hline $\begin{array}{l}\text { 100. Cinnamomum zeylanicum } \\
\text { Blume }\end{array}$ & cinnament (c) & $F, M$ & $\mathrm{D}, \mathrm{G}, \mathrm{T}$ & $B, M$ & D & 0 & $\mathrm{O} / \mathrm{O}$ & 2763 \\
\hline 101. Persea americana Mill. & $\begin{array}{l}\text { sarin, saring, sikya } \\
\text { (u) }\end{array}$ & $\mathrm{F}, \mathrm{M}$ & $\mathrm{D}, \mathrm{J}, \mathrm{L}, \mathrm{O}, \mathrm{W}$ & $B, E, L$ & $\mathrm{D}$ & 0 & $+L^{g}$ & 3356 \\
\hline \multicolumn{9}{|l|}{ LOGANIACEAE } \\
\hline 102. Spigelia anthelmia L. & $\begin{array}{l}\text { bil damaska, biru } \\
\text { damaska (u) }\end{array}$ & M & $\mathrm{P}$ & $\mathrm{P}$ & D & 0 &,$++L^{h}$ & 4438 \\
\hline \multicolumn{9}{|l|}{ LORANTHACEAE } \\
\hline $\begin{array}{l}\text { 103. Struthanthus cassythoides } \\
\text { Millsp. ex Standl. }\end{array}$ & dâwan damaska (u) & M & $A, B, S$ & L,P & $D, P$ & $\mathrm{O}, \mathrm{T}$ & + & 3840 \\
\hline \multicolumn{9}{|l|}{ LYTHRACEAE } \\
\hline $\begin{array}{l}\text { 104. Cuphea mimuloides Cham. } \\
\text { \& Schltr. }\end{array}$ & asu (u) & M & $\mathrm{D}, \mathrm{T}$ & $\mathrm{P}$ & D & 0 & $\mathrm{O} / \mathrm{O}$ & 4054 \\
\hline \multicolumn{9}{|l|}{ MALPIGHIACEAE } \\
\hline $\begin{array}{l}\text { 105. Byrsonima crassifolia (L.) } \\
\text { H.B.K. }\end{array}$ & krabu (u) & $\mathrm{F}, \mathrm{M}, \mathrm{O}$ & $A, D, Q$ & B & D & 0 & + & 2857 \\
\hline $\begin{array}{l}\text { 106. Heteropteris multiflora } \\
\text { (DC.) Hochr. }\end{array}$ & tûsikka (u) & M & $L, Q$ & L & D & 0 & + & 3478 \\
\hline $\begin{array}{l}\text { 107. Hiraea quapara (Aubl.) } \\
\text { Morton }\end{array}$ & dakasa $(\mathrm{u})$ & M & $\mathrm{S}, \mathrm{U}$ & L & D & $\mathrm{T}$ & + & 2335 \\
\hline $\begin{array}{l}\text { 108. Stigmaphyllon pseu- } \\
\text { dopuberum Nied. }\end{array}$ & bil siwanak (u) & M & $Q, V, Z$ & $L, P$ & D & $\mathrm{O}, \mathrm{T}$ & $\mathrm{O} / \mathrm{O}$ & 3793 \\
\hline \multicolumn{9}{|l|}{ MALVACEAE } \\
\hline 109. Hibiscus tiliaceus L. & sani, wahpi (u) & $\mathrm{M}, \mathrm{O}$ & $\mathrm{C}, \mathrm{F}$ & $\mathrm{B}, \mathrm{L}$ & $\mathrm{D}$ & 0 & + & 2185 \\
\hline 110. Sida acuta Burm. L. f. & $\begin{array}{l}\mathrm{k} \text { a t a } \mathrm{r} \text { a } \mathrm{m} \text { a } \mathrm{s}, \\
\text { sakratuni }(\mathrm{u})\end{array}$ & $\mathrm{M}, \mathrm{O}$ & $A, C, W$ & $L, P$ & $\mathrm{D}$ & $\mathrm{O}$ & $+\mathrm{L}^{\mathrm{h}}$ & 3294 \\
\hline $\begin{array}{l}\text { 111. _S. rhombifolia L. alnimuk } \\
\text { (u) }\end{array}$ & muluh, muluh & $\mathrm{M}, \mathrm{O}$ & $A, C, F, L$ & $\mathrm{~L}$ & D & 0 & $+L^{h}$ & 3587 \\
\hline \multicolumn{9}{|l|}{ MELIACEAE } \\
\hline 112._Carapa guianensis Aubl. & saba $(\mathrm{u})$ & $\mathrm{M}, \mathrm{O}$ & $A, F$ & $\mathrm{~B}$ & $\mathrm{D}$ & 0 &,$++L^{g}$ & 4369 \\
\hline 113. Cedrela odorata $\mathrm{L}$. & suyun, winkur (u) & $\mathrm{M}, \mathrm{O}$ & $A, F, Q, T$ & B & D & $\mathrm{O}$ & $-\mathrm{L} / \mathrm{O}$ & 4365 \\
\hline 114. Swietenia macrophylla King & pauluh, yulu & 0 & - & - & - & - & - & 4413 \\
\hline \multicolumn{9}{|l|}{ MORACEAE } \\
\hline $\begin{array}{ll}115 . & \text { Artocarpus altilis } \\
\text { (Parkinson) Fosberg }\end{array}$ & bredpuk (u) & $\mathrm{F}, \mathrm{M}$ & A & $S$ & $P$ & $\mathrm{~T}$ & $\mathrm{O} /+\mathrm{L}^{\mathrm{a}}$ & 3423 \\
\hline 116. Castilla elastica_Sessé & taspul (u) & $\mathrm{F}, \mathrm{M}$ & A & $\mathrm{s}$ & $\mathrm{P}$ & $\mathrm{T}$ & $\mathrm{N}$ & NV \\
\hline $\begin{array}{l}\text { 117. Chlorophora tinctoria (L.) } \\
\text { Gaud. * }\end{array}$ & pan lalahka (u) & 0 & - & - & - & - & - & NV \\
\hline 118. Ficus sp. & taspul (u) & 0 & - & - & - & - & - & NV \\
\hline $\begin{array}{l}\text { 119. Poulsenia armata (Miq.) } \\
\text { Standl. }\end{array}$ & pan yaunaka $(\mathrm{u})$ & $\mathrm{O}$ & - & - & - & - & - & NV \\
\hline
\end{tabular}


WANI

\begin{tabular}{|c|c|c|c|c|c|c|c|c|}
\hline Nombre Gientifico ${ }^{1}$ & Nombre Comun² & Usos $^{3}$ & $\begin{array}{l}\text { Aplicaciones } \\
\text { Medicinales }\end{array}$ & $\begin{array}{l}\text { Materiales } \\
\text { Usados }^{5}\end{array}$ & $\begin{array}{l}\text { Modo de } \\
\text { Preparacion }\end{array}$ & $\begin{array}{l}\text { Modo de } \\
\text { Administra- } \\
\text { cion? }\end{array}$ & $\begin{array}{l}\text { Presencia } \\
\text { Alcaloide/ } \\
\text { glucosido }\end{array}$ & \# Etiqueta $^{9}$ \\
\hline \multicolumn{9}{|l|}{ MYRISTICACEAE } \\
\hline 120. Myristica fragrans Houtt. & nutmeg (c) & $\mathrm{F}, \mathrm{M}$ & G & $\mathrm{F}$ & $\mathrm{D}$ & 0 & $+\mathrm{L}$ & 2753 \\
\hline 121. Virola koschnyi Warb. & pan âwas (u) & $\mathrm{M}, \mathrm{O}$ & $A, D, F$ & $B, L, S$ & $D, P$ & $0, T$ & $+\mathrm{L}$ & 2398 \\
\hline \multicolumn{9}{|l|}{ MYRTACEAE } \\
\hline 122. Psidium guajava $\mathrm{L}$. & kuru, burimak (u) & $\mathrm{F}, \mathrm{M}, \mathrm{O}$ & $D, F, G, H, I, S$ & $\mathrm{~B}, \mathrm{~L}$ & $D, I$ & $\mathrm{~B}, \mathrm{O}$ & $+L^{b}$ & 3443 \\
\hline $\begin{array}{l}\text { 123. Syzygium aromaticum (L.) } \\
\text { Merr. \& Perry }\end{array}$ & cloves (c) & $\mathrm{F}, \mathrm{M}$ & $A, C, G, O$ & C & D & 0 & $\mathrm{O} / \mathrm{O}$ & 4442 \\
\hline \multicolumn{9}{|l|}{ PASSIFLORACEAE } \\
\hline 124._Passiflora biflora_Lam. & waiku pas-bah (u) & M & $\mathrm{F}, \mathrm{I}, \mathrm{K}$ & $L, P$ & D & 0 & $\mathrm{O} / \mathrm{O}$ & 4105 \\
\hline 125. P. quadrangularis $\mathrm{L}$ & wahamtari u) & $F, M$ & $A, F, M, S$ & $\mathrm{~L}$ & $D, J$ & $0, T$ & $+\mathrm{L}^{\mathrm{h}}$ & 3513 \\
\hline \multicolumn{9}{|l|}{ PHYTOLACCACEAE } \\
\hline $\begin{array}{l}\text { 126. Petiveria alliacea L. } \\
\text { sabatkira }(\mathrm{m})\end{array}$ & surua, kiski & M & $A, D, L, O$ & $L, P, R$ & $D, P$ & $0, T$ & $+L^{d}$ & 3959 \\
\hline $\begin{array}{l}\text { 127. Phytolacca rivinoides Kunth } \\
\text { \& Bouché }\end{array}$ & tilba pata $(m)$ & $F, M$ & $E, X$ & $L, R$ & D & 0 & $+\mathrm{L}^{\mathrm{c}}$ & 3261 \\
\hline \multicolumn{9}{|l|}{ PIPERACEAE } \\
\hline $\begin{array}{l}\text { 128. Peperomia pellucida_(L.) } \\
\text { H.B.K }\end{array}$ & sumu yal $(\mathrm{u})$ & M & $\mathrm{B}, \mathrm{I}, \mathrm{V}, \mathrm{W}$ & $P$ & $\mathrm{D}$ & 0 & $\mathrm{O} / \mathrm{O}$ & 3744 \\
\hline 129. P. peltata C. DC. & muih-muih (u) & $\mathrm{M}$ & $B, I, V$ & $\mathrm{P}$ & $\mathrm{D}$ & 0 & $0 / 0$ & 2242 \\
\hline 130. Piper auritum H.B.K. & kalamata (u) & $\mathrm{F}, \mathrm{M}$ & $A, C, F, G$ & $\mathrm{~L}$ & $\mathrm{I}, \mathrm{J}, \mathrm{P}$ & $0, T$ &,$++L^{\mathrm{g}}$ & 2719 \\
\hline $\begin{array}{lrr}131 . & P . & \text { jacquemontianum } \\
\text { (Kunth) DC. } & \\
\end{array}$ & pansan $(\mathrm{u})$ & $M$ & $A, F, G$ & $\mathrm{~L}$ & $B, I$ & $\mathrm{~B}, \mathrm{O}$ & + & 3952 \\
\hline 132. P. peltatum L. & kalamata (u) & $F, M$ & $A, C, F, G$ & $\mathrm{~L}$ & $D, P$ & $B, O, T$ &,$++L^{d}$ & 3928 \\
\hline \multicolumn{9}{|l|}{ POLYGONACEAE } \\
\hline 133. Coccoloba uvifera (L.) L. & waham (u) & $\mathrm{F}, \mathrm{M}$ & $\mathrm{D}, \mathrm{G}, \mathrm{S}$ & $\mathrm{B}, \mathrm{L}$ & $\mathrm{D}$ & 0 & $0 / 0$ & 3445 \\
\hline \multicolumn{9}{|l|}{ PORTULACACEAE } \\
\hline 134. Portulaca oleracea L. & dislah pula (u) & M & $\mathrm{P}$ & $\mathrm{P}$ & $\mathrm{D}$ & 0 & $+L^{b}$ & 4035 \\
\hline \multicolumn{9}{|l|}{ RHIZOPHORACEAE } \\
\hline 135. Rhizophora mangle L. & mankru (u) & $\mathrm{M}, \mathrm{O}$ & $\mathrm{D}, \mathrm{S}$ & B & $\mathrm{D}$ & 0 & $\mathrm{O} /+\mathrm{L}^{\mathrm{a}}$ & 2096 \\
\hline \multicolumn{9}{|l|}{ RUBIACEAE } \\
\hline \multirow[t]{2}{*}{ 136. Alibertia edulis (L. Rich.) } & guayabillo (s) & $F, M$ & $A, C, Q$ & $B, L$ & $\mathrm{D}$ & $0, T$ & $\mathrm{O} / \mathrm{O}$ & 3787 \\
\hline & & & A. Rich. ex DC. & & & & & \\
\hline $\begin{array}{l}\text { 137. Borreria laevis (Lam.) } \\
\text { Griseb. }\end{array}$ & (u) titiska mâ baka & M & $\mathrm{B}, \mathrm{I}, \mathrm{L}, \mathrm{S}, \mathrm{U}$ & L & $D, J, P$ & $\mathrm{~T}$ & + & 3264 \\
\hline $\begin{array}{l}\text { 138. Cinchona pubescens } \\
\text { Vahl }\end{array}$ & quina (s) & $M$ & $F, M$ & $B, M$ & $\mathrm{D}$ & 0 & $+L^{h}$ & 4354 \\
\hline 139. Coffea arabica L. & was baraska (u) & $F, M$ & $F, U$ & $\mathrm{E}$ & $\mathrm{D}, \mathrm{N}$ & $0, T$ & $+L^{f}$ & NV \\
\hline 140. Hamelia patens Jacq. & (u) ${ }^{\text {pauka damaska }}$ & $M$ & $B, F, I, M, S, U$ & $L, P$ & $D, P$ & $0, T$ & + & 2768 \\
\hline & & & & & & & & \\
\hline
\end{tabular}




\begin{tabular}{|c|c|c|c|c|c|c|c|c|}
\hline Nombre Gientifico' & Nombre Gomun² & Usos ${ }^{3}$ & $\begin{array}{l}\text { Aplicaciones } \\
\text { Medicinales }\end{array}$ & $\begin{array}{l}\text { Materiales } \\
\text { Usados }^{5}\end{array}$ & $\begin{array}{l}\text { Modo de } \\
\text { Preparacion }\end{array}$ & $\begin{array}{l}\text { Modo de } \\
\text { Administra- } \\
\text { cion? }\end{array}$ & $\begin{array}{l}\text { Presencia } \\
\text { Alcaloide/ } \\
\text { glucosido }\end{array}$ & \# Etiqueta ${ }^{9}$ \\
\hline $\begin{array}{l}\text { 141. Hemidiodia ocimifolia } \\
\text { (Willd.) Schum. }\end{array}$ & kanbala (u) & M & G & L & D & 0 & + & 3901 \\
\hline $\begin{array}{l}\text { 142. Posoqueria latifolia (Rudge) } \\
\text { Roem. \& Schult. }\end{array}$ & lasap, lasat (u) & $F, M$ & $\mathrm{D}, \mathrm{Q}$ & $\mathrm{B}, \mathrm{L}$ & D & 0 & + & 4314 \\
\hline $\begin{array}{l}\text { 143. Psychotria elata (Sw.) } \\
\text { Hammel }\end{array}$ & pauka kungmak (u) & M & $F, L, M$ & $\mathrm{~F}, \mathrm{~S}$ & $\mathrm{D}$ & $0, T$ & + & 4456 \\
\hline \multicolumn{9}{|l|}{ RUTACEAE } \\
\hline $\begin{array}{ll}\text { 144. } & \text { Citrus aurantifolia } \\
\text { (Christm.) } & \text { Swingle }\end{array}$ & $\begin{array}{l}\text { lîma, limus sapahni } \\
\text { (u) }\end{array}$ & $F, M$ & $C, D, F, G, I, L, P$ & $F, L, R$ & $D, J$ & 0 & $+\mathrm{L}^{\mathrm{c}}$ & 3677 \\
\hline 145. C. paradisi Macfad. & sadik (u) & $F, M$ & $D, F, H$ & $\mathrm{~F}$ & $\mathrm{~J}$ & 0 & $+L^{d}$ & 3681 \\
\hline 146. C. sinensis (L.) Osbeck & aransa $(\mathrm{u})$ & $\mathrm{F}, \mathrm{M}$ & $\mathrm{D}, \mathrm{F}, \mathrm{H}, \mathrm{L}$ & $\mathrm{F}, \mathrm{L}$ & $\mathrm{D}, \mathrm{J}$ & 0 & $+\mathrm{L}^{\mathrm{h}}$ & 4450 \\
\hline \multicolumn{9}{|l|}{ SAPINDACEAE } \\
\hline 147. Melicoccus bijugatus Jacq. & sûnaka (u) & $\mathrm{F}, \mathrm{M}$ & $\mathrm{D}, \mathrm{Q}$ & $\mathrm{E}, \mathrm{L}$ & $\mathrm{D}$ & 0 & $\mathrm{O} / \mathrm{O}$ & 3435 \\
\hline 148. Sapindus saponaria L. & suhnaka $(\mathrm{u})$ & 0 & - & - & - & - & - & 2771 \\
\hline \multicolumn{9}{|l|}{ SAPOTACEAE } \\
\hline 149. Chrysophyllum cainito L. & tinaka $(\mathrm{u})$ & $\mathrm{F}, \mathrm{M}$ & $\mathrm{D}, \mathrm{F}, \mathrm{Q}$ & $\mathrm{F}, \mathrm{L}$ & $\mathrm{D}, \mathrm{N}$ & 0 & $+\mathrm{L}^{\mathrm{e}}$ & 3350 \\
\hline $\begin{array}{l}\text { 150. Manilkara zapota (L) P. } \\
\text { Royen }\end{array}$ & îban, sabakan (u) & $\mathrm{F}, \mathrm{M}$ & $A, S$ & $\mathrm{~S}$ & $P$ & $\mathrm{~T}$ & $+\mathrm{L}^{\mathrm{e}}$ & 2792 \\
\hline $\begin{array}{l}\text { 151. Pouteria sapota (Jacq.) H.E. } \\
\text { Moore \& Stearn }\end{array}$ & $\operatorname{sipul}(u)$ & $\mathrm{F}, \mathrm{M}, \mathrm{O}$ & $A, D, G, S$ & $B, E, L$ & $D, P$ & $0, T$ & $+\mathrm{L}^{\mathrm{h}}$ & 2710 \\
\hline \multicolumn{9}{|l|}{ SCROPHULARIACEAE } \\
\hline $\begin{array}{l}\text { 152. Bacopa procumbens (Mill.) } \\
\text { Greenm. }\end{array}$ & sapaka (u) & M & $A, X$ & $\mathrm{~L}$ & $D, J$ & $0, T$ & $\mathrm{O} / \mathrm{O}$ & 2781 \\
\hline $\begin{array}{l}\text { 153. Lindernia diffusa (L.) } \\
\text { Wettst.ex Dugand \& Jacks }\end{array}$ & $\begin{array}{l}\text { kuntitir, tipismak } \\
\text { (u) }\end{array}$ & M & $x$ & $P$ & D & 0 & + & 2728 \\
\hline 154._Scoparia dulcis L. & $\begin{array}{l}\text { ubitna salalaini, } \\
\text { ubitna bikisni }(\mathrm{u})\end{array}$ & M & $\mathrm{B}, \mathrm{C}, \mathrm{T}, \mathrm{W}$ & $L, P, R$ & D & 0 &,$++L^{h}$ & 3501 \\
\hline \multicolumn{9}{|l|}{ SIMAROUBACEAE } \\
\hline 155._Quassia amara L. & batakka dî basta (u) & M & $\mathrm{B}, \mathrm{F}, \mathrm{M}, \mathrm{T}$ & B & $\mathrm{D}$ & 0 &,$++L^{h}$ & 4353 \\
\hline \multicolumn{9}{|l|}{ SOLANACEAE } \\
\hline 156. Capsicum annuum_var. & anmak (u) & $F, M$ & $A, L, S$ & $E, F, L$ & $\mathrm{D}, \mathrm{N}$ & $0, T$ & $+\mathrm{L}^{\mathrm{h}}$ & 4330 \\
\hline \multicolumn{9}{|l|}{$\begin{array}{l}\text { glabriusculium_(Dunal) Heiser \& } \\
\text { Pickersgill }\end{array}$} \\
\hline 157. C. chinensis Jacq. & angmak, anmak (u) & $\mathrm{F}, \mathrm{M}$ & $A, L, S$ & $E, F, L$ & $\mathrm{D}, \mathrm{N}$ & $0, T$ & + & 3605 \\
\hline 158. C. frutescens L. & angmak, anmak (u) & $F, M$ & $\mathrm{I}, \mathrm{L}$ & $\mathrm{F}, \mathrm{L}$ & $\mathrm{D}, \mathrm{J}$ & $0, T$ & $+\mathrm{L}^{\mathrm{h}}$ & 2748 \\
\hline 159. Nicotiana tabacum L. & aka (u) & $\mathrm{M}, \mathrm{O}$ & $A, B$ & $\mathrm{~L}$ & $\mathrm{~N}$ & $0, T$ & $+\mathrm{L}^{\mathrm{h}}$ & NV \\
\hline 160. Physalis angulata $\mathrm{L}$. & $\begin{array}{l}\text { paumak makdasi } \\
(\mathrm{u})\end{array}$ & M & $F, I, M$ & $L, P$ & 1 & 0 & $+\mathrm{L}^{\mathrm{h}}$ & 3695 \\
\hline 161. Solanum lycopersicum L. & paumak (u) & $\mathrm{F}, \mathrm{M}$ & $s$ & $\mathrm{~L}$ & $\mathrm{~J}$ & $\mathrm{~T}$ & $+\mathrm{L}^{\mathrm{h}}$ & 2831 \\
\hline 162. S. tuberosum L. & pai (u) & $\mathrm{F}, \mathrm{M}$ & $\mathrm{G}$ & $\mathrm{R}$ & $\mathrm{J}$ & 0 & $+\mathrm{L}^{\mathrm{h}}$ & NV \\
\hline \multicolumn{9}{|l|}{ STERCULIACEAE } \\
\hline $\begin{array}{l}\text { 163. Melochia villosa (Mill.) } \\
\text { Fawc. \& Rendle }\end{array}$ & kalsa (u) & M & $A, G$ & $\mathrm{~L}$ & D & 0 & + & 4127 \\
\hline 164._Theobroma bicolor H.B.K. * & kuru (u) & $\mathrm{M}, \mathrm{F}$ & $\mathrm{N}$ & $\mathrm{E}$ & $\mathrm{J}$ & $\mathrm{T}$ & + & 4468 \\
\hline 165. T. cacao L. & kakay $(\mathrm{m})$ & $\mathrm{F}, \mathrm{M}$ & $\mathrm{S}, \mathrm{U}$ & $E, L$ & $\mathrm{P}$ & $\mathrm{T}$ & $+\mathrm{L}^{\mathrm{h}}$ & 2815 \\
\hline \multicolumn{9}{|l|}{ TILIACEAE } \\
\hline $\begin{array}{l}\text { 166. Heliocarpus donnell-smithii } \\
\text { Rose * }\end{array}$ & sulduih (u) & $\mathrm{M}, \mathrm{O}$ & s & B & D & $\mathrm{T}$ & O/O & 4466 \\
\hline \multicolumn{9}{|l|}{ TURNERACEAE } \\
\hline 167. Turnera odorata $\mathrm{L}$. & lalahka (u) & M & $A, L, T$ & L & D & $\mathrm{O}$ & $\mathrm{O} / \mathrm{O}$ & 2760 \\
\hline
\end{tabular}


WANI

\begin{tabular}{|c|c|c|c|c|c|c|c|c|}
\hline Nombre Gientifico' ${ }^{1}$ & Nombre Gomun² & Usos $^{3}$ & $\begin{array}{l}\text { Aplicaciones } \\
\text { Medicinales }\end{array}$ & $\begin{array}{l}\text { Materiales } \\
\text { Usados }^{5}\end{array}$ & $\begin{array}{c}\text { Modo de } \\
\text { Preparacion }\end{array}$ & $\begin{array}{l}\text { Modo de } \\
\text { Administra- } \\
\text { cion? }\end{array}$ & $\begin{array}{l}\text { Presencia } \\
\text { Alcaloide/ } \\
\text { glucosido }\end{array}$ & \# Etiqueta ${ }^{9}$ \\
\hline 168. T. ulmifolia L. & udu pulu (u) & M & $A, F, L, X$ & $\mathrm{~L}$ & $\mathrm{D}$ & 0 & $+\mathrm{L}^{\mathrm{h}}$ & 3887 \\
\hline \multicolumn{9}{|l|}{ VERBENACEAE } \\
\hline 169. Avicennia germinans (L.) L. & mankru baraska $(\mathrm{u})$ & $\mathrm{M}, \mathrm{O}$ & $\mathrm{D}, \mathrm{Q}$ & B & D & 0 & O/O & 2824 \\
\hline 170. Cornutia pyramidata L. * & mâ baka sikka (u) & $M$ & $\mathrm{~F}, \mathrm{~S}$ & $L, R$ & $\mathrm{D}$ & $0, T$ & $\mathrm{O} / \mathrm{O}$ & 4276 \\
\hline 171. Lantana camara L. & $\begin{array}{l}\text { makdasi was } \\
\text { baraska }(\mathrm{u})\end{array}$ & M & s & L & $D, P$ & $\mathrm{~T}$ & $\mathrm{O} /+\mathrm{L}^{\mathrm{c}}$ & NV \\
\hline 172. L. trifolia L. & wî wî (u) & $M$ & A & $\mathrm{L}$ & $P$ & $\mathrm{~T}$ & $\mathrm{O} /+\mathrm{L}^{\mathrm{c}}$ & 995 \\
\hline $\begin{array}{l}\text { 173. Lippia alba (Mill.) N.E. Br. } \\
\text { ex Britton \& Wilson }\end{array}$ & tubaruka (u) & $F, M$ & $\mathrm{C}, \mathrm{F}, \mathrm{I}, \mathrm{W}$ & L & $\mathrm{D}, \mathrm{I}$ & 0 & $\mathrm{O} /+\mathrm{L}^{\mathrm{c}}$ & 3908 \\
\hline 174. L. micromera Schauer & wî wing (u) & $M$ & $\mathrm{C}, \mathrm{G}, \mathrm{I}, \mathrm{L}, \mathrm{W}$ & $\mathrm{L}$ & $D, I$ & 0 & $\mathrm{O} /+\mathrm{L}^{\mathrm{c}}$ & 2779 \\
\hline 175. Phyla nodiflora (L.) Greene & saya dî basta (u) & M & $R, S$ & $P$ & $D, P$ & $0, T$ & $+L^{g}$ & 4436 \\
\hline $\begin{array}{l}\text { 176. Stachytarpheta cayennensis } \\
\text { (Rich.) Vahl. }\end{array}$ & $\begin{array}{l}\text { dî bastsa batakka } \\
\text { (u) }\end{array}$ & $M$ & $F, G, P, X$ & L & D & 0 & $+L^{c}$ & 3551 \\
\hline 177. S. jamaicensis_(L.) Vahl. & dî basta batakka (u) & M & $F, G, P, X$ & $\mathrm{~L}$ & $\mathrm{D}$ & 0 & $+\mathrm{L}^{\mathrm{g}}$ & 3628 \\
\hline 178. Tamonea spicata Aubl. & kiaya (u) & $F, M$ & $\mathrm{~F}, \mathrm{G}, \mathrm{L}$ & $\mathrm{L}$ & $\mathrm{D}$ & 0 & + & 4163 \\
\hline 179. Vitex kuylenii Standl. & sasanika (u) & 0 & - & - & - & - & - & 2299 \\
\hline \multicolumn{9}{|l|}{ VOCHYSIACEAE } \\
\hline $\begin{array}{l}\text { 180. Vochysia ferruginea G. } \\
\text { Martens }\end{array}$ & pan was $(\mathrm{u})$ & 0 & - & - & - & - & - & 2880 \\
\hline \multicolumn{9}{|l|}{$\begin{array}{l}\text { LILIOPSIDA } \\
\text { (MONOCOTILEDONEAS) }\end{array}$} \\
\hline \multicolumn{9}{|l|}{ ALOEACEAE } \\
\hline 181. Aloe vera $\mathrm{L}$. & singwanaka luih $(\mathrm{u})$ & M & $\mathrm{B}, \mathrm{N}, \mathrm{S}$ & $\mathrm{L}$ & J & $0, T$ & $+L^{g}$ & 2743 \\
\hline \multicolumn{9}{|l|}{ ARACEAE } \\
\hline $\begin{array}{l}\text { 182. Colocasia esculenta (L.) } \\
\text { Schott }\end{array}$ & pailumakka (u) & $\mathrm{F}$ & - & - & - & - & - & 2788 \\
\hline $\begin{array}{l}\text { 183. Xanthosoma sagittifolium } \\
\text { (L.) Schott }\end{array}$ & wilis (u) & $\mathrm{F}$ & - & - & - & - & - & 2791 \\
\hline \multicolumn{9}{|l|}{ ARECACEAE } \\
\hline $\begin{array}{l}\text { 184. Acoelorraphe wrightii } \\
\text { (Griseb. \& H. Wendl.) H. Wendl. } \\
\text { ex Becc. }\end{array}$ & papta (u) & $\mathrm{M}, \mathrm{O}$ & $\mathrm{D}$ & $R$ & $\mathrm{D}$ & 0 & $\mathrm{O} / \mathrm{O}$ & 2782 \\
\hline 185. Bactris gasipaes Kunth & siuli, supa (u) & $\mathrm{F}, \mathrm{M}, \mathrm{O}$ & G & $\mathrm{F}$ & $\mathrm{D}$ & 0 & $\mathrm{O} / \mathrm{O}$ & 2772 \\
\hline $\begin{array}{l}\text { 186. Calyptrogene ghiesbre- } \\
\text { ghtiana (Linden \& H.Wendl.) H. } \\
\text { Wendl. }\end{array}$ & $\begin{array}{l}\text { kataramah umah } \\
\text { (u) }\end{array}$ & 0 & - & - & - & - & - & NV \\
\hline 187. Cocos nucifera $\mathrm{L}$. & anau, annu, annah (u) & $\mathrm{F}, \mathrm{M}, \mathrm{O}$ & $D, P$ & $\mathrm{~F}$ & $\mathrm{D}, \mathrm{I}$ & 0 & $+L^{c}$ & NV \\
\hline 188. Elaeis guineensis_Jacq. & auhka (u) & $\mathrm{F}, \mathrm{M}, \mathrm{O}$ & $x$ & $\mathrm{~F}$ & $\mathrm{D}$ & 0 & $\mathrm{~N} / \mathrm{O}$ & NV \\
\hline 189. E. oleifera (Kunth) Cortés & auhka (u) & $\mathrm{F}, \mathrm{M}, \mathrm{O}$ & $x$ & $\mathrm{~F}$ & $\mathrm{D}$ & 0 & $\mathrm{~N} / \mathrm{O}$ & NV \\
\hline $\begin{array}{l}\text { 190. Raphia taedigera_(C. Mart.) } \\
\text { C. Mart. }\end{array}$ & siliku (u) & $\mathrm{O}$ & - & - & - & - & - & NV \\
\hline \multicolumn{9}{|l|}{ BROMELIACEAE } \\
\hline 191. Ananas comosus (L.) Merr. & masa, mâsahti (u) & $\mathrm{F}$ & - & - & - & - & - & 2727 \\
\hline 192. Bromelia pinguin L. & ahsi, wakari (u) & $\mathrm{O}$ & - & - & - & - & - & 2737 \\
\hline \multicolumn{9}{|l|}{ COMMELINACEAE } \\
\hline 193. Commelina erecta L. & waswas (u) & M & $\mathrm{s}$ & $L, M$ & J & $\mathrm{T}$ & $\mathrm{O} / \mathrm{O}$ & 4344 \\
\hline \multicolumn{9}{|l|}{ CYPERACEAE } \\
\hline 194. Cyperus luzulae_(L.) Retz. & kapalak (u) & $\mathrm{M}, \mathrm{O}$ & $\mathrm{D}$ & $R$ & $\mathrm{D}$ & 0 & $\mathrm{O} / \mathrm{O}$ & 3687 \\
\hline 195._Kyllinga tibialis Ledeb. & $\begin{array}{l}\text { karasmak damaska } \\
\text { (u) }\end{array}$ & M & $\mathrm{F}$ & $R$ & D & 0 & + & 4114 \\
\hline
\end{tabular}




\begin{tabular}{|c|c|c|c|c|c|c|c|c|}
\hline Nombre Gientifico 1 & Nombre Gomun² & Usos $^{3}$ & $\begin{array}{l}\text { Aplicaciones } \\
\text { Medicinales }\end{array}$ & $\begin{array}{l}\text { Materiales } \\
\text { Usados }^{5}\end{array}$ & $\begin{array}{l}\text { Modo de } \\
\text { Preparacion }\end{array}$ & $\begin{array}{c}\text { Modo de } \\
\text { Administra- } \\
\text { cion? }\end{array}$ & $\begin{array}{l}\text { Presencia } \\
\text { Alcaloide/ } \\
\text { glucosidlo }\end{array}$ & \# Etiqueta ${ }^{9}$ \\
\hline $\begin{array}{l}\text { 196. Rhynchospora barbata } \\
\text { (Vahl.) Kunth }\end{array}$ & prisisi (u) & 0 & - & - & - & - & - & 2635 \\
\hline 197. R. ciliata Vahl. & prisisi (u) & 0 & - & - & - & - & - & 4199 \\
\hline \multicolumn{9}{|l|}{ DIOSCOREACEAE } \\
\hline 198._Dioscorea trifida_L. & usi (u) & $\mathrm{F}$ & - & - & - & - & - & 2844 \\
\hline \multicolumn{9}{|l|}{ HAEMODORACEAE } \\
\hline 199. Xiphidium caeruleum Aubl. & $\begin{array}{l}\text { swilawan, umah } \\
\text { tikbus (u) }\end{array}$ & M & $A, B, S, W$ & L & D & $\mathrm{O}, \mathrm{T}$ & $\mathrm{O} /+\mathrm{L}^{\mathrm{d}}$ & 2806 \\
\hline \multicolumn{9}{|l|}{ LILIACEAE } \\
\hline 200. Allium cepa L. & onyan $(\mathrm{u})$ & $F, M$ & $L, P$ & $\mathrm{R}$ & $\mathrm{J}$ & 0 & $\mathrm{~N}$ & NV \\
\hline 201. Allium sativum L. & kyalic (m) & $\mathrm{F}, \mathrm{M}$ & $\mathrm{A}, \mathrm{H}, \mathrm{P}$ & $\mathrm{R}$ & $\mathrm{J}$ & 0 & $\mathrm{~N}$ & NV \\
\hline \multicolumn{9}{|l|}{ MUSACEAE } \\
\hline 202. Musa_sp. & yâmanh (u) & $\mathrm{F}, \mathrm{M}, \mathrm{O}$ & $B, D, U$ & $\mathrm{~F}, \mathrm{~S}$ & $\mathrm{~N}, \mathrm{P}$ & $\mathrm{O}, \mathrm{T}$ & $+\mathrm{L}^{\mathrm{g}}$ & NV \\
\hline 203. M. paradisiaca L. & waka, waki (u) & $\mathrm{F}, \mathrm{M}, \mathrm{O}$ & $B, D, U$ & $\mathrm{~F}, \mathrm{~S}$ & $\mathrm{~N}, \mathrm{P}$ & $0, T$ & $+\mathrm{L}^{\mathrm{g}}$ & NV \\
\hline $\begin{array}{l}\text { 204. M. paradisiaca var. } \\
\text { sapientum (L.) Kuntze }\end{array}$ & $\begin{array}{l}\text { inkini, ingkinih, } \\
\text { pasa, wakisa (u) }\end{array}$ & $\mathrm{F}, \mathrm{M}, \mathrm{O}$ & $B, D, U$ & $\mathrm{~F}, \mathrm{~S}$ & $\mathrm{~N}, \mathrm{P}$ & $\mathrm{O}, \mathrm{T}$ & $+\mathrm{L}^{\mathrm{g}}$ & NV \\
\hline \multicolumn{9}{|l|}{ POACEAE } \\
\hline $\begin{array}{l}\text { 205. Andropogon leucostachyus } \\
\text { H.B.K. }\end{array}$ & wâlang (u) & 0 & - & - & - & - & - & 2693 \\
\hline 206. Axonopus compresus (Sw.) & wâlang (u) & 0 & - & - & - & - & - & 4200 \\
\hline \multicolumn{9}{|l|}{ P. Beauv. } \\
\hline 207. A. poiophyllus_Chase & wâlang (u) & 0 & - & - & - & - & - & 2615 \\
\hline $\begin{array}{l}\text { 208. Bambusa vulgaris Schrad. } \\
\text { ex Wendl. }\end{array}$ & itikna (u) & $\mathrm{O}$ & $D, F$ & $\mathrm{R}$ & D & $\mathrm{O}$ & $+L^{g}$ & 2711 \\
\hline 209. Coix lacryma-jobi L. & am minik, am mak (u) & $\mathrm{M}, \mathrm{O}$ & $\mathrm{A}, \mathrm{I}, \mathrm{S}$ & $E, R$ & $\mathrm{D}$ & 0 & $+L^{a}$ & 2646 \\
\hline $\begin{array}{l}\text { 210. Cymbopogon citratus } \\
\text { (Nees) Stapf }\end{array}$ & ti (u) & $F, M$ & $\mathrm{~F}, \mathrm{G}, \mathrm{L}$ & $\mathrm{L}$ & 1 & 0 & $+L^{g}$ & 3682 \\
\hline $\begin{array}{l}\text { 211. Dichanthelium sphaero- } \\
\text { carpon var. floridanum (Vasey) } \\
\text { Davidse }\end{array}$ & wâlang (u) & 0 & - & - & - & - & - & 2641 \\
\hline $\begin{array}{l}\text { 212. Gynerium sagittatum } \\
\text { (Aubl.) P. Beauv. }\end{array}$ & dapa (u) & $\mathrm{M}, \mathrm{O}$ & $\mathrm{B}, \mathrm{I}, \mathrm{S}$ & $\mathrm{R}$ & D & $\mathrm{O}$ & $\mathrm{O} / \mathrm{O}$ & 3870 \\
\hline $\begin{array}{l}\text { 213. Leptocoryphium lanatum } \\
\text { (H.B.K.) Nees }\end{array}$ & wâlang (u) & 0 & - & - & - & - & - & 2625 \\
\hline 214. Mesosetum blakei_Swallen & wâlang (u) & 0 & - & - & - & - & - & 2639 \\
\hline 215. Olyra latifolia L. & wâlang (u) & $\mathrm{M}$ & $\mathrm{s}$ & $L, R$ & $\mathrm{D}$ & $T$ & $\mathrm{O} / \mathrm{O}$ & 2495 \\
\hline 216. Oryza sativa L. & rais $(\mathrm{u})$ & $\mathrm{F}$ & $\mathrm{D}, \mathrm{S}$ & $\mathrm{E}$ & $\mathrm{B}, \mathrm{I}$ & $\mathrm{B}, \mathrm{O}$ & $+\mathrm{L}^{\mathrm{h}}$ & 2756 \\
\hline 217. Panicum maximum Jacq. & wâlang sikka (u) & 0 & - & - & - & - & - & 2759 \\
\hline 218. P. mertensii Roth & wâlang (u) & 0 & - & - & - & - & - & 2538 \\
\hline 219. P. pilosum Sw. & wâlang (u) & 0 & - & - & - & - & - & 2494 \\
\hline 220. P. purpurascens Raddi & wâlang para (u) & 0 & - & - & - & - & - & 2761 \\
\hline 221. Saccharum officinarum L. & tisnak (u) & $\mathrm{F}, \mathrm{M}, \mathrm{O}$ & $\mathrm{D}, \mathrm{I}, \mathrm{L}, \mathrm{S}$ & $\mathrm{L}, \mathrm{M}$ & $\mathrm{D}, \mathrm{J}$ & O,T & $-\mathrm{L} / \mathrm{O}$ & 2764 \\
\hline 222. Zea mays L. & am, ama (u) & $\mathrm{F}, \mathrm{M}, \mathrm{O}$ & 1 & C & $\mathrm{D}$ & 0 & $+\mathrm{L}^{\mathrm{h}}$ & 2766 \\
\hline \multicolumn{9}{|l|}{ SMILACACEAE } \\
\hline 223. Smilax spinosa Mill. & $\begin{array}{l}\text { samalai wasalana- } \\
\text { ka (u) }\end{array}$ & M & $B, S, T$ & $\mathrm{R}$ & D & 0 & $\mathrm{O} /+\mathrm{L}^{\mathrm{c}}$ & 3820 \\
\hline \multicolumn{9}{|l|}{ ZINGIBERACEAE } \\
\hline 224. Curcuma longa L. * & azáfran (s) & $\mathrm{M}, \mathrm{O}$ & $\mathrm{s}$ & $\mathrm{R}$ & $\mathrm{J}$ & $\mathrm{T}$ & $\mathrm{O} /+\mathrm{L}^{\mathrm{c}}$ & 4467 \\
\hline 225. Zingiber officinale Roscoe & sinsa, marid puluni $(u)$ & $\mathrm{F}, \mathrm{M}$ & $\mathrm{D}, \mathrm{F}, \mathrm{G}, \mathrm{L}$ & $\mathrm{R}$ & $\mathrm{D}$ & 0 & $\mathrm{O} /+\mathrm{L}^{\mathrm{a}}$ & 2826 \\
\hline
\end{tabular}

